Article

\title{
Analysis of the Hydrodynamic Effects of Gas Permeation in a Pilot-Scale Fluidized Bed Membrane Reactor
}

\author{
Chenxi Bai ${ }^{1}$, Yao Xiao ${ }^{1}$, Ruifeng Peng ${ }^{1}$, John R. Grace ${ }^{2}$ and Yumin Chen ${ }^{1, *}$ \\ 1 School of Electrical Power Engineering, China University of Mining and Technology, Xuzhou 221116, China; \\ ts16130011a3@cumt.edu.cn (C.B.); ts16130013a3@cumt.edu.cn (Y.X.); ts18130112p31@cumt.edu.cn (R.P.) \\ 2 Department of Chemical and Biological Engineering, University of British Columbia, 2360 East Mall, \\ Vancouver, BC V6T 1Z3, Canada; john.grace@ubc.ca \\ * Correspondence: yuminchen@cumt.edu.cn; Tel.: +86-0516-83592000; Fax: +86-0516-83590140
}

Received: 10 November 2018; Accepted: 19 December 2018; Published: 25 December 2018

\begin{abstract}
This study experimentally investigates the effects of gas extraction/addition, via multiple vertical membrane panels, on the hydrodynamics in different regions of a pilot-scale gas fluidized bed membrane reactor (FBMR), based on differential pressure signals measured at different vertical bed sections at high temperature. In a bed section where membrane panels were installed and activated, the extraction of gas caused the average bubble size to increase, but decreased the number of smalland medium-sized bubbles. This effect of gas extraction penetrated into bed sections above the active membrane panel, but attenuated with increasing distance away from the extraction location. The attenuation rate was much faster in FBMR with lower bed voidage, mainly due to the large decrease of the drag force exerted by gas extraction on fluidizing gas in a denser bed. With the same inlet gas velocity, gas addition favored the growth of bubbles, especially in the upper bed sections compared with operation without gas permeation. The increase of the effective fluidizing velocity was the major reason for the increase of the bubble size during gas addition. These findings preliminarily suggest that membrane units should not be installed in or below fast-reacting zones in a scale-up FBMR, and operation with a lower bed voidage is preferable to avoid the formation of large bubbles enhanced by gas extraction.
\end{abstract}

Keywords: fluidization; membrane reactor; pressure measurement; gas permeation; hydrodynamics

\section{Introduction}

Fluidized bed membrane reactors (FBMRs) have gained increasing interest as one of the promising technologies to facilitate process intensification, by combining the selective removal of reaction products or the addition of reactant via membranes with the advantages of fluidized bed reactors. With the assistance of membranes, higher product yields and selectivity are possible under safer operation conditions. Nevertheless, inefficiency issues associated with hot spot formation, large pressure drops, and concentration polarization could be avoided or improved [1-3].

Dense metal membranes have been widely used for product in situ separation, especially, for ultra-pure hydrogen production by hydrocarbon reforming in fluidized bed reactors. Partil et al. [4], Chen et al. [5], Andrés et al. [3], Roses et al. [1] and Arratibel et al. [6] experimentally investigated the subject of hydrogen production in a FBMR by steam or autothermal reforming of methane, with in situ removal of hydrogen via Pd-based dense membranes. $\mathrm{H}_{2}$ permselective membranes also have been used in a fluidized bed reactor to promote the hydrogen yield in the water-gas shift reaction [2], steam reforming of higher hydrocarbons [7] and dehydrogenation reaction [8]. By integrating hydrogen 
separation with reforming reactions, higher reactant conversions were achieved at lower temperatures, and ultra-pure hydrogen flow with purity $>99.995 \%$ were obtained in the permeate lines [2-7]. Another interesting application of membranes is the selective dosing of reactant-mostly oxygen-into a chemical reactor. Similar operations, such as distribution of secondary air can also be facilitated by using porous ceramic membranes or side ports instead of selective membranes. The distribution of the oxidative reactant on the membrane was experimentally found to reduce the unwanted side reactions [9], improve yields [10], and benefit the safe operation with a wide range of reactant compositions, mainly due to the possible proper alteration of the concentration profile along the reactor by this method. Consequently, axial gas back-mixing was efficiently decreased [11-13], and the interphase mass transfer could be enhanced [14].

Despite the demonstrated advantages of integrating the membrane processes with a fluidized bed reactor, there are still several issues hindering the industrial application of FBMRs. For example, the efficiency of membrane units usually suffers serious deterioration in practical operations [5]. Also, the stability of the membrane in long-term operation at high temperature is far from satisfactory [2]. The fundamental principles for reasonable arrangements of membranes according to the reactor configuration, scale, and process design are not yet clear [10]. All the aforementioned challenges greatly depend on understanding the hydrodynamics in FBMRs. For example, the overall permeation rate of membrane units is mainly determined by the contacting efficiency of the membrane with target gases [3,15], which is significantly related to the dynamic distribution of the emulsion phase in the bed. The interaction between particles and membranes, which is closely associated with the dynamic movements of the solids, is one of major factors affecting the thermal and chemical stability of membranes [2]. Additionally, the bubble-to-emulsion phase mass transfer limitation also tends to become more pronounced in a FBMR $[1,10]$. Variation in the volumetric flowrate caused by gas extraction or addition could affect the pressure drop in the bed [7]. Therefore, research on the hydrodynamic effects of the membrane processes, especially the interaction of gas extraction/addition with in-bed hydrodynamics, is required to improve the performance of FBMRs. Although correlative studies focusing on this subject are still few, it has been attracting more and more attention as an emerging issue.

The hydrodynamic effects of gas extraction/addition on flow dynamics in a FBMR are closely related to operational factors, such as fluidization velocity, permeation flux, bed materials and operating temperature/pressure. Moreover, the change of configuration, location and arrangements of the membranes makes these interactions more complex. For example, formation of a densified layer, variation of bubble properties and gas back-mixing have preliminarily been investigated, but the experimental results reported by different researchers are inconsistent. De Jong et al. [16] and Dang et al. [17] found that stagnant/densified zones readily formed near the membranes during gas extraction with flat membranes, which usually also acted as reactor walls, and would be improved by increasing the extraction fraction. On the other hand, Wassie et al. [18,19] experimentally demonstrated that the formation of a stable densified zone in the bed while extracting gas via flat vertical membranes only occurred with smaller particles, especially at high extraction rates. No densified layers were observed during gas extraction when Geldart B type particles $(400 \sim 600 \mu \mathrm{m})$ were used as bed material in experiments conducted by Helmi et al. [20]. In addition, with flat membranes installed, both a reduction [20-22] and a slight increase [16] in the average bubble size were observed during gas extraction from the fluidized bed. Also, Deshmukh et al. [11] experimentally demonstrated that the distributive feeding of oxygen via porous membranes decreased axial gas back-mixing, while the addition of gas through flat membranes was found to increase the gas back-mixing [23]. As mentioned above, the dynamics of gas-solid flow in a membrane-assisted fluidized bed reactor varies with reactor scale, membrane configuration, membrane location, and bed material. A fundamental understanding of the hydrodynamic interaction of the membrane-fluidized bed is still lacking. Accordingly, a deeper insight into the hydrodynamic interaction between gas extraction and gas-solid flow in a FBMR is greatly needed. 
Different experimental techniques, such as particle image velocimetry, digital image analysis, electrical capacity, magnetic resonance tomography and X-ray analysis [19], have been successfully applied to investigate the solid hold-up or bubble properties in lab-scale FBMRs. Min et al. [24] and Wassie et al. [25] found that gas permeation exerted extensive influence on the flow dynamics in a FBMR, rather than only in the zones nearby the activated membrane. Moreover, the effects of gas permeation on the hydrodynamics in different regions of a FBMR were also different $[18,26]$. As a result, more measurement points or larger detection regions are required, which would greatly increase the measurement cost, in order to properly determine the hydrodynamic effects of gas permeation in a scaled-up FBMR. In this case, pressure measurements would be particularly preferred for analyzing the hydrodynamic effects of gas permeation in a scaled-up FBMR with multiple membranes installed at different locations. Pressure measurement could successfully keep its spatial and temporal resolution by increasing the measurement points and sampling frequency, when applied to a scaled-up FBMR. Besides this benefit, pressure measurement not only can provide information about the flow dynamics in a local bed, but also in the other regions at a specified instant $[27,28]$. Accordingly, it is a powerful tool to detect the synergistic effects of gas permeation via different membranes installed in a FBMR. Differential pressure fluctuations are closely correlated with the bubble dynamics between measurement intervals. Thus, they can be favorably applied to characterize the hydrodynamics in different parts of a pilot-scale FBMR [29-31]. With the proper resolution of the differential pressure signals by wavelet analysis or other multi-resolution methods, the multiscale dynamics of gas-solid flows could be adequately investigated $[29,30]$.

This study aims to investigate the hydrodynamic effects of gas permeation on the flow dynamics in a pilot-scale FBMR. Five flat vertical membrane panels were inserted into the bed along the height of the reactor. The effects of gas extraction/addition on the hydrodynamics in different parts of the FBMR were thoroughly studied based on the differential pressure fluctuations. These differential pressure signals were properly resolved by wavelet decomposition to characterize the behaviors of multiscale structures during the gas extraction/addition process. The effects of the gas extraction/addition fraction, permeation location, and bed material on the interactions between gas permeation and gas-solid flow were also studied. The whole study would provide useful proofs to understand how gas permeation behaviors affect the local/global hydrodynamics in a FBMR.

\section{Experimental Setup and Procedure}

A schematic of the gas extracting/adding experiment system is shown in Figure 1. The main cross-section of the stainless steel column is a circle with an internal diameter of $48 \mathrm{~mm}$. Five porous panels made from steel powders, with a dimension of $162 \mathrm{~mm} \times 36 \mathrm{~mm} \times 6 \mathrm{~mm}$ (height $\times$ width $x$ thickness), were installed along the height of the reactor to act as membrane. The distributor was doughnut-shaped, with six equally-spaced holes drilled on the inner side. These holes pointed inward and downwards at an angle of $45^{\circ}$ to the vertical, to reduce the back-pouring of particles into the plenum chamber. Using this type of distributor, stable fluidization has been experimentally demonstrated to be established in FBMRs $[3,5,31]$. The reactor was heated inside by 4 cable heaters and outside the bed by 10 band heaters and 6 strip heaters. The temperature was measured at 8 vertical positions inside the bed by K-type thermocouples. Signals from the thermocouples were sent to a control system to adjust and maintain the required temperature during experiments. Compressed air at room temperature, controlled by pressure regulators and mass flow controllers, was used as fluidizing gas, as well as permeation gas. During the air addition tests (campaign I), compressed air was distributed to different membrane panels at the set flowrates through the permeation lines. In cases of air extraction (campaign II), a vacuum connected to a buffer was started to maintain the driving forces for gas permeating out from the reactor, and the extraction rates in each permeation line were also controlled individually by a mass flow controller, as shown in Figure 1. 


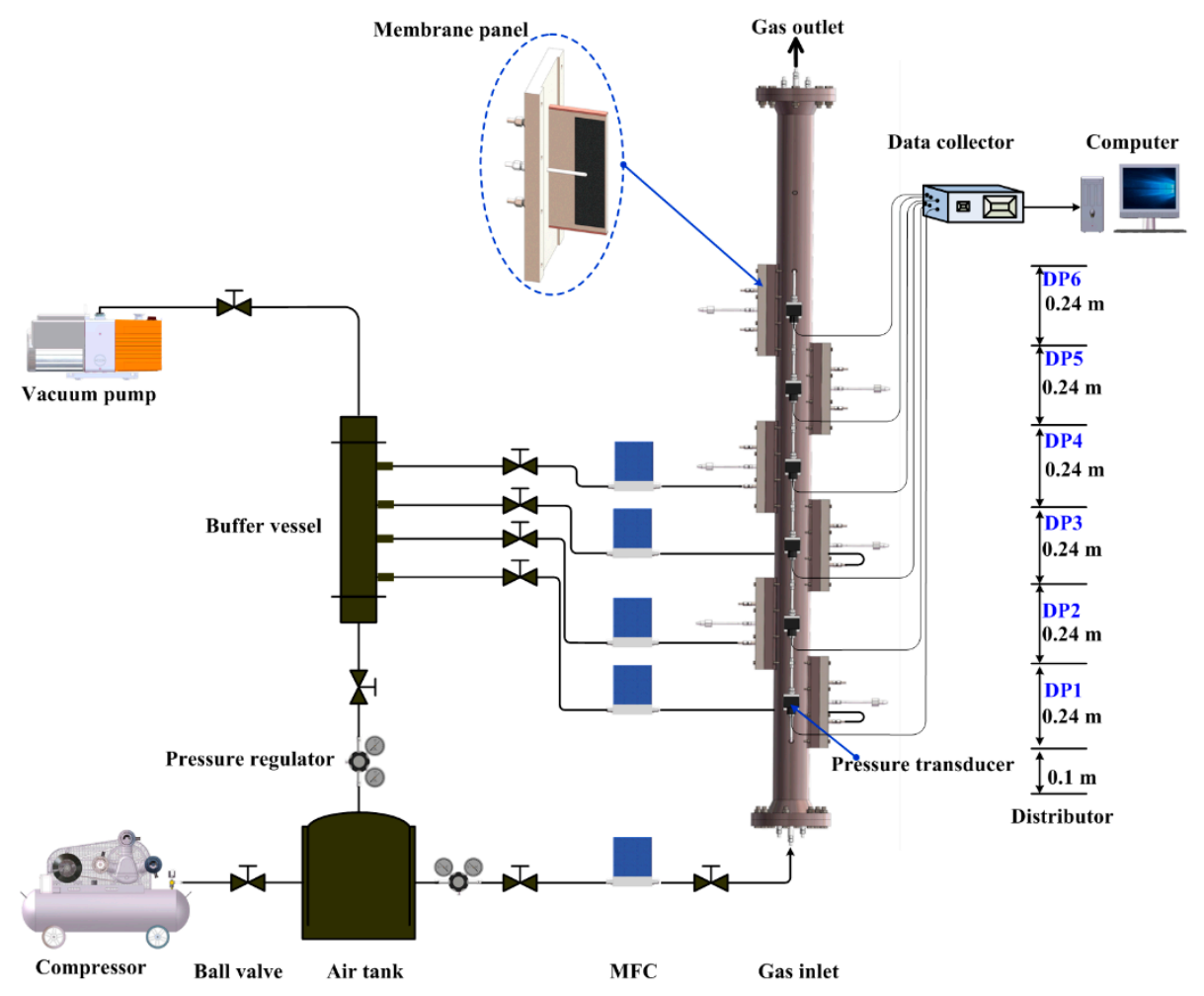

Figure 1. Scheme of the experimental system.

Differential pressure fluctuations, in the form of voltage signals, at five vertical locations were recorded using rapid-response pressure transducers (142PC01D5V, Omega, Surrey, UK). The arrangement of the probe ports for pressure transducers, as well as the locations of the installed porous membranes, are illustrated in Figure 1. Each pressure port was located in the center of the column between two membrane panels, and covered by a porous stainless steel screen which was welded onto the end of the probe to prevent blockage by fine particles. The porous screen was purged with compressed air before starting a new experiment to minimize the damping-effect. The extended steel tubes connecting the probe leg and pressure transducer were cooled by water jackets during the gas-extraction tests. Voltage signals from the pressure transducers were logged into a computer via an A/D converter with 12-bit resolution. A total of 10,000 data points were acquired at a sampling frequency of $100 \mathrm{~Hz}$.

Two different types of particles were tested in this work, and the properties of these particles are listed in Table 1 . The minimum fluidization velocity $\left(U_{m f}\right)$ was determined from the evolution of the pressure drop across the particles bed while decreasing the inlet gas velocity from 40 to $0.5 \mathrm{~L} / \mathrm{min}$. This method has also been used by other researchers to measure $U_{m f}$. In all experiments, the initial bed height was kept at around $1.28 \mathrm{~m}$. For the convenience of the analysis, we called the bed section, where the porous membrane panels were installed and activated, the local bed section. Membrane panels were denoted as membranes \#1-5 according to their position. The permeation line of membrane \#5 was shut off during all tests. Operations without gas extraction/addition here were referred as normal operations. The operation conditions of experiments performed in this study are listed in Table 2. The highest gas extracting velocity of $0.036 \mathrm{~m} / \mathrm{s}$ has been reported during the hydrogen permeation test operated at a hydrogen feed pressure of $2.6 \mathrm{MPa}$ and permeation temperature of $400{ }^{\circ} \mathrm{C}$ [32]. Therefore, the permeation rates employed in this work were from $0.5-3.0 \mathrm{~L} / \mathrm{min}$, which correspond to gas permeation velocities from $0.002 \sim 0.012 \mathrm{~m} / \mathrm{s}$, and were in the same order of magnitude as those reported for the existing hydrogen permselective membranes. 
Table 1. Properties of particulate materials.

\begin{tabular}{cccc}
\hline Material & Diameter Range $(\mathbf{m m})$ & Particle Density, $\varrho_{\mathbf{p}}\left(\mathbf{k g} / \mathbf{m}^{\mathbf{3}}\right)$ & $\mathbf{U}_{\mathbf{m f}}(\mathbf{m} / \mathbf{s})$ \\
\hline Calcined dolomite & $0.18-0.212$ & 1450 & 0.041 \\
Silica sand & $0.18-0.212$ & 2520 & 0.069 \\
\hline
\end{tabular}

Table 2. Operational conditions of experiments performed.

\begin{tabular}{ccccc}
\hline Campaign & Membranes Activated & Bed Material & Permeation Rate (L/min) & $U(\mathbf{m} / \mathbf{s})$ \\
\hline Ref. 1 & 0 & Calcined dolomite & 0 & $0.004-0.37$ \\
Ref. & 0 & Silica sand & 0 & $0.004-0.37$ \\
$\# 1-0$ & Membrane \#1-4 & Calcined dolomite & 5,10 & $0.09-0.14$ \\
$\# 1-1$ & Membrane \#1 & Calcined dolomite & $1.5,3.0$ & $0.09-0.14$ \\
$\# 1-2$ & Membrane \#2 & Calcined dolomite & $1.5,3.0$ & $0.09-0.14$ \\
$\# 1-3$ & Membrane \#3 & Calcined dolomite & $1.5,3.0$ & $0.09-0.14$ \\
$\# 1-4$ & Membrane \#1-4 & Silica sand & 5,10 & $0.09-0.14$ \\
$\# 1-5$ & Membrane \#1 & Silica sand & $1.5,3.0$ & $0.09-0.14$ \\
$\# 1-6$ & Membrane \#2 & Silica sand & $1.5,3.0$ & $0.09-0.14$ \\
$\# 1-7$ & Membrane \#3 & Silica sand & $1.5-3.0$ & $0.09-0.14$ \\
$\# 2-0$ & Membrane \#1-4 & Calcined dolomite & -5 & $0.09-0.14$ \\
$\# 2-1$ & Membrane \#1 & Calcined dolomite & $-0.5,-1.0,-1.5$ & $0.09-0.14$ \\
$\# 2-2$ & Membrane \#2 & Calcined dolomite & $-0.5,-1.0,-1.5$ & $0.09-0.14$ \\
$\# 2-3$ & Membrane \#3 & Calcined dolomite & $-0.5,-1.0,-1.5$ & $0.09-0.14$ \\
$\# 2-4$ & Membrane \#1-4 & Silica sand & -5 & $0.09-0.14$ \\
$\# 2-5$ & Membrane \#1 & Silica sand & $-1.0,-1.5$ & $0.09-0.14$ \\
$\# 2-6$ & Membrane \#2 & Silica sand & $-1.0,-1.5$ & $0.09-0.14$ \\
$\# 2-7$ & Membrane \#3 & Silica sand & $-1.0,-1.5$ & $0.09-0.14$ \\
\hline
\end{tabular}

Notes: (1) Symbol minus " -" before the permeation rate means extraction; (2) refs. 1 and 2 campaigns represent normal operations; (3) the other operational conditions: $T=550^{\circ} \mathrm{C}, P=0.1 \mathrm{MPa}, H_{\text {ini }}=1.28 \mathrm{~m}$.

\section{Methods of Analysis}

\subsection{Statistical Analysis}

The passage of bubble through the measuring interval can be properly registered by differential pressure measurements. According to Equation (1), which has been widely used to estimate the pressure drop across the fluidized bed, the average fluctuation amplitude is proportional to the average bubble size $[29,30]$ :

$$
\Delta P=\left[\rho_{p}(1-\varepsilon)+\rho_{f} \mathcal{\varepsilon}\right] g H
$$

The amplitude of a time series of pressure signal, $x_{i}(I=1,2,3, \ldots, N)$, is expressed by the standard deviation as:

$$
\sigma=\sqrt{\frac{1}{N-1} \sum_{i=1}^{N}\left(x_{i}-x_{a v}\right)^{2}}, x_{a v}=\frac{1}{N} \sum_{i=1}^{N} x_{i}
$$

where $N$ is the number of data points in pressure signal $x$. In this study, a relative fluctuation amplitude derived by normalizing the standard deviation $\sigma$ with the average $x_{a v}$, expressed as $\sigma_{r}=\sigma / x_{a v}$, was used to characterize the evolution of the average bubble size under the operational conditions.

\subsection{Frequency Domain Analysis}

A discrete Fourier transform algorithm was used to convert the measured signals from the time domain into the frequency domain. The major frequency content of the pressure fluctuations in a gas fluidized bed reactor is normally below $10 \mathrm{~Hz}$, so that sampling at $100 \mathrm{~Hz}$ is sufficient to capture most flow features [33]. The power spectrum of the measured signals was calculated by the non-parametric 
method. In order to decrease variations, the power spectrum was obtained by averaging a number of sub-spectra. Thus, the time series was divided into $L$ segments of individual length $N_{s}$, represented as:

$$
x_{i}=x\left(n+i N_{s}\right) n=1,2, \ldots, L
$$

The power-spectrum of each segment is calculated as:

$$
P_{x x}^{i}(f)=\frac{1}{N_{s} U}\left|\sum_{n=1}^{N_{s}} x_{i}(n) w(n) \exp (-j 2 \pi f n)\right|^{2}
$$

where $U$ is a normalizing factor representing power in the window function $w(n)$, and can be expressed as:

$$
U=\frac{1}{N_{s}} \sum_{n=1}^{N_{s}} w^{2}(n)
$$

The averaged power spectrum is then calculated by:

$$
P_{x x}(f)=\frac{1}{L} \sum_{i=1}^{L} P_{x x}^{i}(f)
$$

The frequency resolution of the power spectrum is given by $\Delta f=f_{s} / N_{s}$, where $f_{s}$ is the sampling frequency; 8192 points of the data series in each run were used for the Fourier transform giving a frequency resolution of $0.012 \mathrm{~Hz}$. With each run repeated 9 times, a smooth power spectral was obtained by averaging the data sets. A Hanning window [33] has been used as window function $w(n)$ in this study, and can be expressed as:

$$
\mathrm{w}(n)=\frac{1}{2}\left(1-\cos \left(\frac{2 \pi n}{N-1}\right)\right)
$$

\subsection{Discrete Wavelet Transform (DWT)}

Wavelet transform separates a signal into multi-resolution components, so the fine and coarse resolution components capture different features in the signals. It has been referred to as a sort of mathematical microscope, with different parts of the signal examined by automatically adjusting the focus. The discrete wavelet transform (DWT) has been commonly used in multi-scale complex system resolution due to its inherent time-level locality characteristics [34]. The DWT is defined as:

$$
\operatorname{DWT}(l, k)=\frac{1}{\sqrt{2^{l}}} \int X(t) \psi\left(\frac{t-k \cdot 2^{l}}{2^{l}}\right) d t
$$

In Equation (5), $X(t)$ is a differential pressure time series, $\psi$ is mother wavelet function, $l$ and $k$ represent the resolution level and time lag coefficient, respectively. The DWT decomposes the discrete signal, $X(n)$, into an ordered set of orthogonal approximation and detailed functions, $A^{j}(n)$ and $D^{j}(n)$, as expressed by the following equations:

$$
\begin{aligned}
& A^{l+1}(n)=\sum_{m} h_{m-2 n} A^{l}(m) \\
& D^{l+1}(n)=\sum_{m} g_{m-2 n} D^{l}(m)
\end{aligned}
$$

where $h_{n}$ and $g_{n}$ represent a low-pass filter and a high-pass filter, respectively, and vary with the mother wavelets function used. The original data are considered to be the level function coefficients at the highest level, denoted as $A_{0}(t)$. Thus, the original signal was decomposed into two components $A_{1}(t)$ 
and $D_{1}(t)$ at the first level, and the decomposition of $A_{l}(t)$ continues until the desired decomposition level $L$ was reached. Subsequently, the original signal can be recovered by summing the approximation and details as follows:

$$
X(t) \approx A_{L}(t)+D_{L}(t)+D_{L-1}(t)+\cdots D_{1}(t)=\sum_{1}^{L} D_{l}(t)+A_{L}(t)
$$

The detailed and approximation components, $D_{l}(t)$ and $A_{L}(t)$, lie within a frequency band of $\left[2^{-(l+1)} f_{s}, 2^{-l} f_{s}\right]$ and $\left[0,2^{-(l+1]} f_{s}\right]$, respectively, where $f_{s}$ is the sampling frequency. In the present study, Daubechies wavelets were used as mother wavelet functions for the resolution of differential pressure signals [30]. The decomposition error between the original signals and reconstructed signals reached its minimum at compact support 2 for the decomposition levels 1-10, so that a compact support 2 was employed in wavelet transform. With Daub2 as the mother wavelet function, the amount of entropy remaining in $A_{L}$ approximated zero at decomposition level 8 for all signal studied, suggesting a resolution limit of 8 levels for this work.

A very useful parameter derived from wavelet decomposition is wavelet energy or power, which can be calculated as:

$$
\begin{aligned}
& E_{l}^{A}=\sum_{t=1}^{N}\left|A_{l}(t)\right|^{2} \\
& E_{l}^{D}=\sum_{t=1}^{N}\left|D_{l}(t)\right|^{2}
\end{aligned}
$$

The total energy of the original signal $X(t)$ is obtained by summing energies of the sub-signals, including $A^{L}(t)$ and $D^{l}(t)(l=1,2, \ldots, L)$, and then the energy fraction of $X(t)$ distributed in each frequency band (each level of the signal) can be written as:

$$
E f_{D}^{l}=E_{l}^{D} /\left(\sum_{l=1}^{L} E_{l}^{D}+E_{L}^{A}\right)
$$

\section{Results and Discussion}

The minimum fluidization velocity, $U_{m f}$, in FBMR with five porous membrane panels installed, but with permeation lines shut off, were measured experimentally in this study by recording the pressure drop across the bed as the inlet flowrate decreases from 40 to $0.5 \mathrm{~L} / \mathrm{min}$. $U_{m f}$ is defined as the point where the pressure drop across the particles bed stops increasing linearly with increasing gas velocity, and remains constant after this critical velocity. As listed in Table $1, U_{m f}$ is equal to $0.041 \mathrm{~m} / \mathrm{s}$ $(4.5 \mathrm{~L} / \mathrm{min})$ and $0.069 \mathrm{~m} / \mathrm{s}(7.5 \mathrm{~L} / \mathrm{min})$ for calcined dolomite and silica sand, respectively.

The effects of gas extraction/addition on the variation of $\sigma_{r}$ along the height of the FBMR, with membranes \#1-4 activated are shown in Figure 2. In normal operations without membrane activation, $\sigma_{r}$ increases along with the bed height, indicating continual growth of the average bubble size. When membranes \#1-4 are activated for gas extraction, $\sigma_{r}$ remarkably increases and remains relatively stable until a decrease was observed at the measurement intervals of DP5. Whether for operations with an inlet velocity of $0.09 \mathrm{~m} / \mathrm{s}$ or $0.14 \mathrm{~m} / \mathrm{s}$, the evolution tendency of $\sigma_{r}$ is similar, which indicates that gas extraction has pronounced effects on the bubble properties in the bed. Gas addition through membranes \#1-4, with a membrane arrangement similar to that of the gas extraction tests, led to increased $\sigma_{r}$, and the incrementation was augmented at higher bed sections and higher permeation rates. 


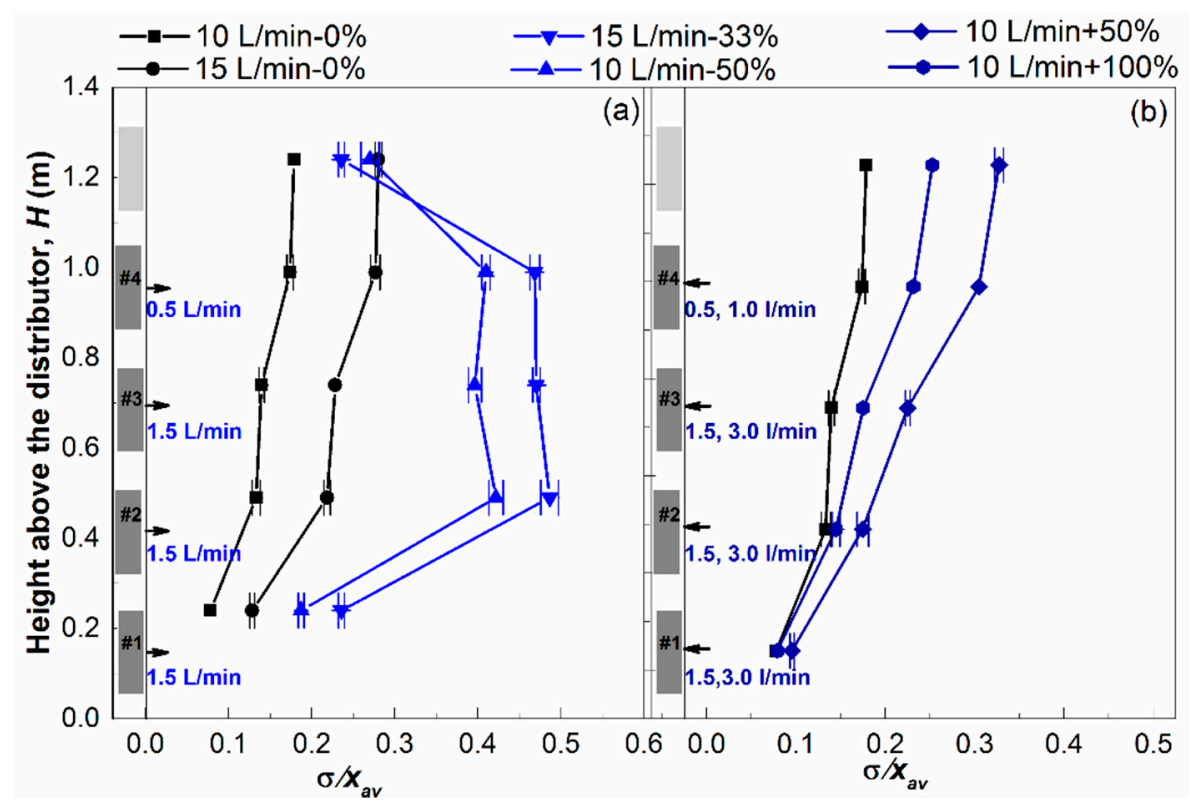

Figure 2. Effects of (a) gas extraction and (b) gas addition through porous membranes on the $\sigma_{r}$ of the differential pressure signals. The other operation conditions: $T=550{ }^{\circ} \mathrm{C}, P=0.1 \mathrm{MPa}, H_{i n i}=1.28 \mathrm{~m}$, calcined dolomite.

\subsection{Effects of Gas Extraction}

The changes of the $\sigma_{r}$ while extracting gas out of the bed at three different locations are shown in Figure 3, with the extraction fraction varying from 5 to $15 \%$. The inlet superficial gas velocity was $0.09 \mathrm{~m} / \mathrm{s}$, and calcined dolomite particles were used as bed material. A remarkable increase in the $\sigma_{r}$ was observed for signals measured in the local bed section. Gas extraction also led to an increase of the $\sigma_{r}$ of the pressure fluctuations measured in the bed sections above the active membrane panels. However, this effect generally decreased with the distance of the measurement location from the extracting locations. For example, while extracting gas out of the bed from membrane \#2 at a rate of $1.5 \mathrm{~L} / \mathrm{min}$, the $\sigma_{r}$ at DP2 increased from 0.11 to 0.33 , but the incrementation of the $\sigma_{r}$ at DP3 decreased to 0.14 , and was almost reduced to zero at DP5, as can be seen in Figure 3. Compared to regions above the extraction locations, the bed section below the active membrane panel seemed hardly to be affected by gas extraction, as indicated by only a slight increase of the $\sigma_{r}$ at DP1 and DP2 with the activation of membranes \#2 and \#3, respectively. According to studies by Julián et al. [22], gas extraction through an immersed tubular membrane or a reactor-wall membrane were also found to exert minor influences on the bubble properties in the region below the permeation zone in a two-section two-zone fluidized bed membrane reactor (TS-TZFBMR). 


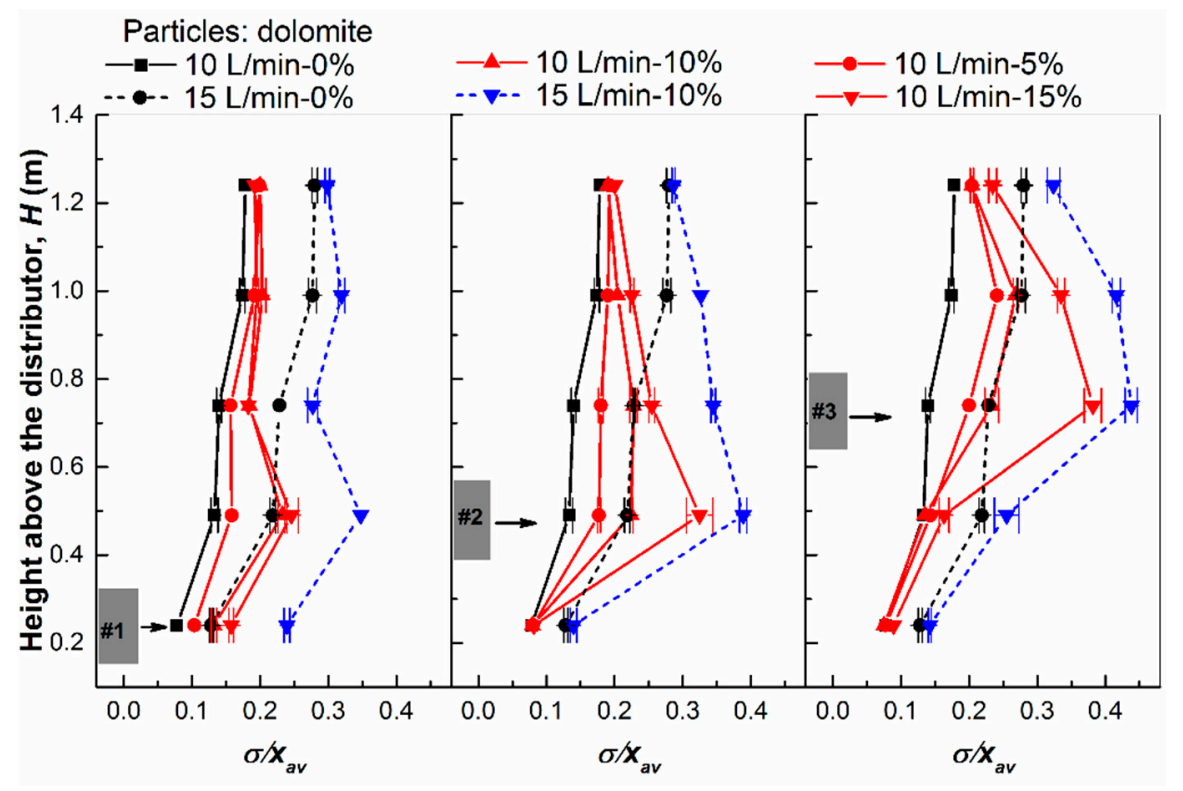

Figure 3. Effects of gas extraction on the $\sigma_{r}$ of the differential pressure signals with the individual activation of membranes \#1-3. The other operation conditions were: $T=550{ }^{\circ} \mathrm{C}, P=0.1 \mathrm{MPa}, H_{\text {ini }}=$ $1.28 \mathrm{~m}, U=0.09-0.14 \mathrm{~m} / \mathrm{s}$, extraction rate $=0.5 \sim 1.5 \mathrm{~L} / \mathrm{min}$, calcined dolomite.

The power spectra of the differential pressure signals measured at DP1, DP2 and DP3 (local bed sections), with gas extracted from membrane \#1, \#2 and \#3, respectively, at a rate of $1.5 \mathrm{~L} / \mathrm{min}$, are illustrated in Figure 4. For comparison, the power spectra for the normal operations are also shown. The main peaks on the spectrum of the differential pressure measured in the gas extraction tests showed much higher power density compared with the signals measured under normal operational conditions, which meant that the size of large bubbles passing through the local bed sections was increased. This result was consistent with analysis based on the evolution of the $\sigma_{r}$. Moreover, the main peaks of the power spectrum were shifted to lower frequency regions, and the power density of minor peaks at higher frequency regions was greatly reduced. As can be seen in Figure 4, for the differential pressure measured at DP2, the main peak of the power spectrum was localized in the frequency region from $0.9 \sim 1.7 \mathrm{~Hz}$ at normal operational conditions, while another major peak appeared at the frequency region from $0.1 \sim 0.4 \mathrm{~Hz}$ in the case of gas extraction. A similar change was also displayed in the power spectra of the differential pressure measured at DP3. The pressure fluctuations with low frequency but high magnitude were mainly excited by large bubbles (voids), while the contents with medium magnitude were related to bubble coalescence/integration and medium size bubbles [30,33]. As can be seen in Figure 4, gas extraction was supposed to enhance the coalescence of bubbles in the local bed section, thus the main frequency of the main peak decreased (number of bubbles decreased due to bubble coalescence), but the peak intensity greatly increased (size of the bubbles increased due to bubble integration). De Jong et al. [16] also experimentally found that gas extraction through flat membranes on the side walls led to increased bubble size in the local section of a fluidized bed reactor. Indeed, as indicated by their experimental results, the incrementation of the equivalent bubble size due to gas extraction also decreased gradually at higher axial position, but not as remarkably as in this study. Wassie et al. [25] reported that the average bubble diameter increased, while the bubble number decreased in the area where active membrane plates were installed, in particular when a high extraction fraction of $40 \%$ was employed. Glass beads with a density of $2500 \mathrm{~kg} / \mathrm{m}^{3}$ and mean particle size of $190 \mu \mathrm{m}$ were used as the bed medium. 


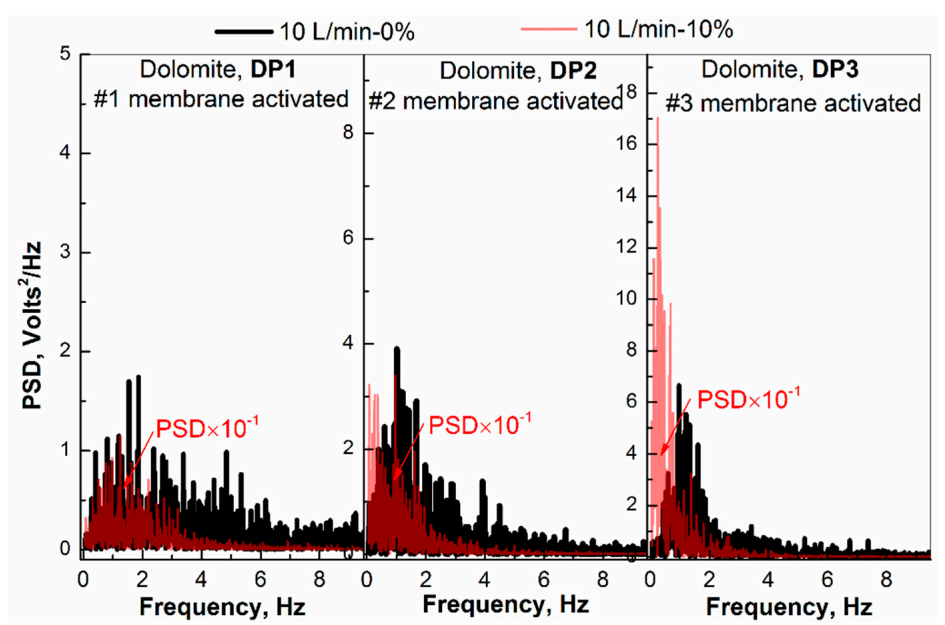

Figure 4. Comparison of the power spectra of the differential pressure in the local bed section measured at the gas extraction and normal operational conditions with the individual activation of membrane \#1 3 for gas extraction. The other operational conditions were: $T=550{ }^{\circ} \mathrm{C}, P=0.1 \mathrm{MPa}, H_{\text {ini }}=1.28 \mathrm{~m}$, $U=0.09 \mathrm{~m} / \mathrm{s}$, extraction rate $=1.5 \mathrm{~L} / \mathrm{min}$, calcined dolomite.

According to the results shown in Figure 3, not only were the differential pressure fluctuations from the local bed section affected by gas extraction, but also other regions in the bed. Figure 5 presents a comparison of the power spectra of the differential pressure measured during the gas extraction tests and normal operation, from the upward and downward bed sections rather than the local bed section. When membrane \#2 (in the DP2 section) was activated for gas extraction, the main frequency of the power spectrum for the signal from DP3 (up bed section) was shifted from 1.3 to $1.1 \mathrm{~Hz}$, and the peak intensity was significantly increased. However, the power spectrum of the differential pressure from DP1 (downward bed section) did not vary greatly compared with the normal operation, and only the main peak intensity increased slightly. Similar results were also observed for the gas extraction with the activated membrane \#3, as shown in Figure 5. Therefore, it is reasonable to conclude that gas extraction in a FBMR not only greatly affected the hydrodynamics in the local bed section, but also in the deeper bed section, especially in regions above the extraction location.

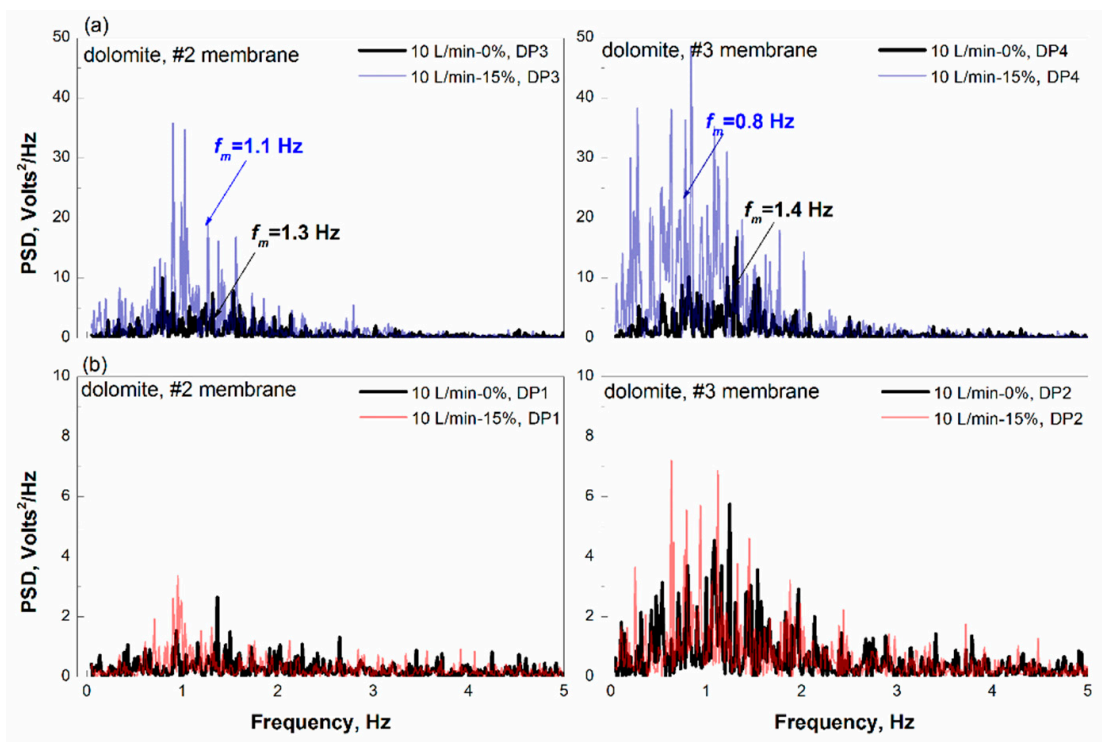

Figure 5. Comparison of the power spectra of the differential pressure in (a) the upward bed section and $(\mathbf{b})$ the downward bed section measured at gas extraction and normal operational conditions. The other operational conditions were: $T=550^{\circ} \mathrm{C}, P=0.1 \mathrm{MPa}, H_{\text {ini }}=1.28 \mathrm{~m}, U=0.09 \mathrm{~m} / \mathrm{s}$, extraction rate $=1.5 \mathrm{~L} / \mathrm{min}$, calcined dolomite. 
In fact, these effects of gas extraction exerted on the flow dynamics were closely related to the distance between the extraction location and the investigated zones. The power spectra of the differential pressure measured in the bed section DP5, with activated membranes \#1, \#2 and \#3, are compared in Figure 6. As illustrated in Figure 1, membrane \#1 is mostly far away from DP5, and so is membrane \#2, while membrane \#3 is just adjacent to DP5. Compared with the normal operation, the increase in the main peak intensity of the power spectra was the largest while extracting gas with activated membrane \#3, but such an increase was not so evident with activated membrane \#1, in which case only a minor shift of the peak frequency is observed in the power spectrum. This finding confirmed the proposed conjecture that the effects of gas extraction by the membrane unit on the hydrodynamics in FBMR decreased with the distance from the extracting location. According to the above analysis, when arranging multiple membrane units in a large FBMR, the membrane units should not be installed in or below fast-reacting zones, otherwise large bubbles induced by gas extraction would greatly decrease the overall reaction efficiency.

The energy distribution at different levels of the differential pressure signal, which were decomposed using Daub2 during multi-resolution analysis, are presented in Figure 7. Gas was extracted out of the bed via membrane \#1-3, separately, at an inlet gas velocity of $0.09 \mathrm{~m} / \mathrm{s}$, with calcined dolomite as bed material, as shown in Figure 7. For normal operation, the maximum energy fraction of the differential pressure signals was occupied by D4 $(3.125 \sim 6.25 \mathrm{~Hz})$ at DP1 (the bottom bed), and gradually shifted to lower-frequency sub-signals at a higher bed section, for example to D6 $(0.78 \sim 1.56 \mathrm{~Hz})$ level sub-signals at DP4. D4 was likely caused by behaviors of the medium-sized bubbles, while D6 was closely related to large-sized bubbles [35]. The change of the wavelet energy distribution illustrated well the evolution of small and medium-sized bubbles towards large-sized bubbles along the height of the bed. Basically, the formation of large bubbles was enhanced by bubble growth (excess gas entering the bubble) and coalescence of small bubbles [36].

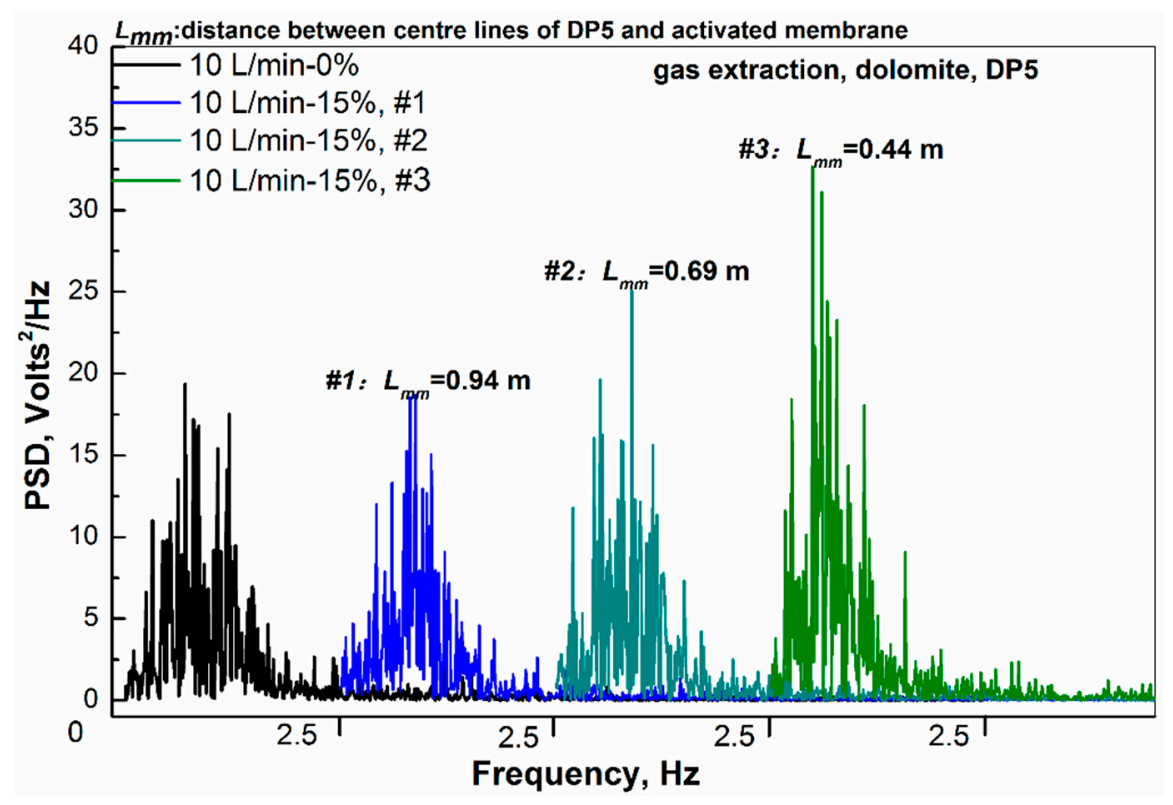

Figure 6. Effects of the extraction location on the power spectra of the differential pressure measured at DP5. The other operational conditions were: $T=550{ }^{\circ} \mathrm{C}, P=0.1 \mathrm{MPa}, H_{\text {ini }}=1.28 \mathrm{~m}, U=0.09 \mathrm{~m} / \mathrm{s}$, extraction rate $=1.5 \mathrm{~L} / \mathrm{min}$, calcined dolomite. 

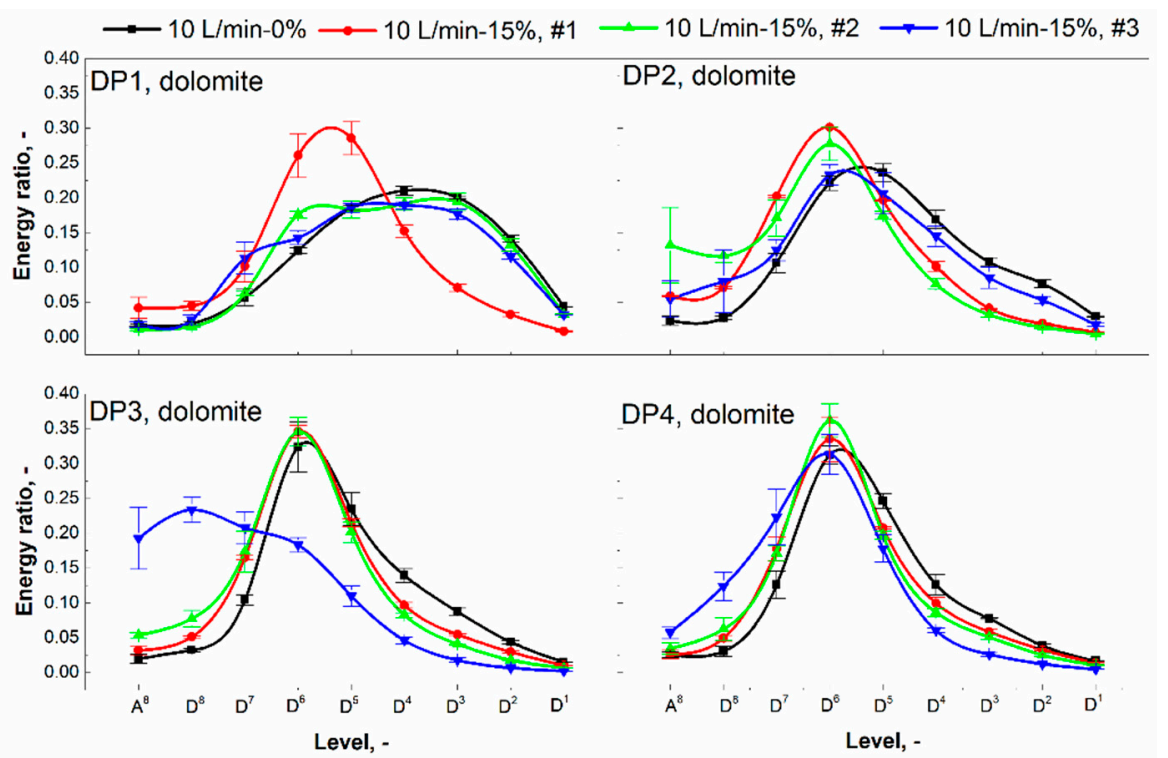

Figure 7. Effects of the gas extraction location on the energy distribution profiles of multi-scale resolved differential pressure signals from DP1 DP4. The other operational conditions were: $T=550{ }^{\circ} \mathrm{C}, P=$ $0.1 \mathrm{MPa}, H_{\text {ini }}=1.28 \mathrm{~m}, U=0.09 \mathrm{~m} / \mathrm{s}$, extraction rate $=1.5 \mathrm{~L} / \mathrm{min}$, calcined dolomite.

Gas extraction remarkably decreased the energy distributed in the high-frequency sub-signal regions (D5-D2, 1.56 25 Hz), and increased the energy fraction in the low-frequency sub-signal regions (A8-D7, 0 1.56 Hz), especially for signals measured in the local bed section. As illustrated in Figure 7, for the differential pressure measured at DP2, the energy fraction of the D8 $(0.195 \sim 0.39 \mathrm{~Hz})$ level sub-signal region was increased from 0.012 to 0.13 , while the energy fraction of the D3 $(3.25 \sim 6.5 \mathrm{~Hz})$ level sub-signal was reduced from 0.14 to 0.046 during the extraction test with the activated membrane \#2. This variation suggested that gas extraction enhanced the formation of structures with larger size (lower frequency) in the local bed sections, compared with the normal operation. Due to the effects of gas extraction, the energy fraction of the A8-D8 $(0 \sim 0.195 \mathrm{~Hz})$ level sub-signal regions greatly increased and became the main components of the differential pressure signals from DP3 with activated membrane \#3. A8-D8 was supposed to be caused by large structures at the scale of the reactor [34,35], such as slugs or large voids which should be avoided in a gas-solid fluidized bed reactor. The reasons for the formation of macro-scale structures in the DP3 section rather than in the DP1 or DP2 sections were that at normal operations, the average bubble size in DP3 already was larger than that in the lower bed sections due to bubble calescence and growth [36].

The results presented in Figure 7 showed that the variation caused by gas extraction was also observed on the wavelet energy distribution of the differential pressure signals from the upward bed sections, for example, from DP2 DP4 with activated membrane \#1, from DP3 DP4 with activated membrane \#2. However, the difference between the wavelet energy distribution of the signals measured from the gas extraction tests and normal operation decreased as the distances of measurement locations from the active membrane increased. This analysis result confirmed the assumption proposed above.

\subsection{Effects of Gas Addition}

The variances of the $\sigma_{r}$ along with the bed height when adding gas via membranes \#1 3 at an inlet gas velocity of $0.09 \mathrm{~m} / \mathrm{s}$, with calcined dolomite as bed material are shown in Figure 8 . In our studies, the primary inlet gas velocity was kept the same $(0.09 \mathrm{~m} / \mathrm{s}$ or $0.14 \mathrm{~m} / \mathrm{s})$ for normal operation during the gas addition tests, so the total gas flowrate in the bed actually increased in the local bed section and upper regions. Gas addition slightly increased the $\sigma_{r}$ at the local bed section, and the incrementation continually increases at higher bed locations, which differed greatly from the evolution of the $\sigma_{r}$ during the gas extraction tests, as shown in Figure 2. Christensen et al. [12,13] and Dang et al. [17] 
experimentally investigated the effects of gas addition on the bubble properties in the fluidized bed, but they maintained the total flowrate, which was mainly the result of keeping the inlet gas and, secondarily, the added gas flowrate, constant during all the experiments. Thus, reduced average bubble sizes were observed in their studies.

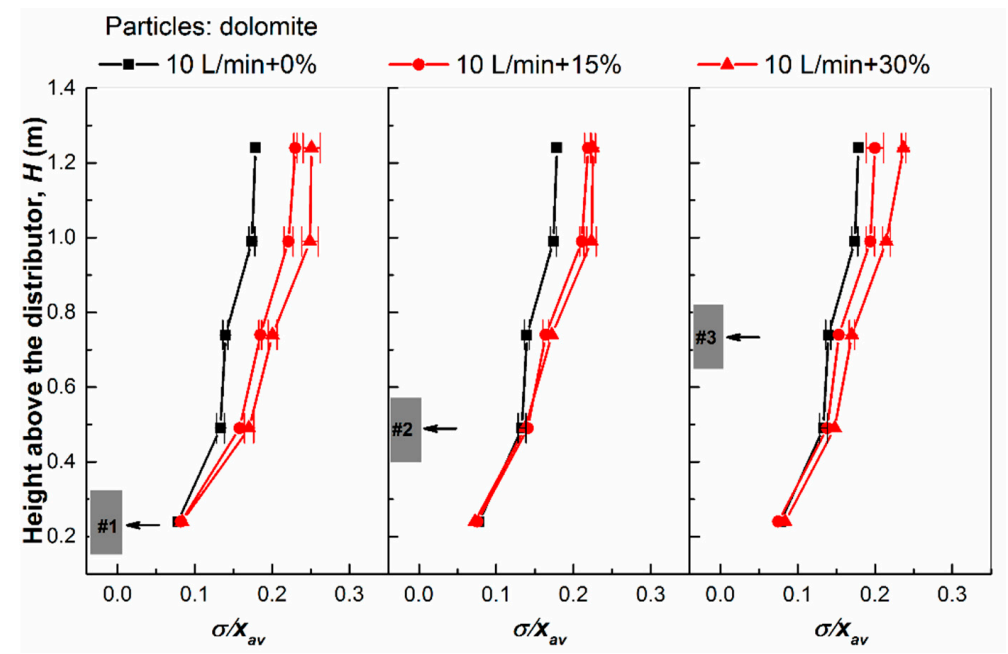

Figure 8. Effects of gas addition through porous membranes on the $\sigma_{r}$ of the differential pressure signals. The other operational conditions were: $T=550{ }^{\circ} \mathrm{C}, P=0.1 \mathrm{MPa}, H_{\text {ini }}=1.28 \mathrm{~m}, U=0.09 \mathrm{~m} / \mathrm{s}$, addition rate $=1.5 \sim 3.0 \mathrm{~L} / \mathrm{min}$, calcined dolomite.

The effects of gas addition on the power spectra of the differential pressure measured in the local bed section are illustrated in Figure 9. Compared with the normal operation, the main peak intensity of the power spectra displayed a slight increase with the addition of gas to the bed via the specified porous membrane panel. An increase of the power density at the frequency bands $>2 \mathrm{~Hz}$ was observed in all cases investigated, as shown in Figure 9. In the upper bed sections above the addition location, the incrementation of the main peak intensity became larger, and would be more significant at higher bed sections, as shown in Figure 10. However, the power density distribution patterns were similar for tests with or without gas addition in the upper bed sections, for example in DP5.

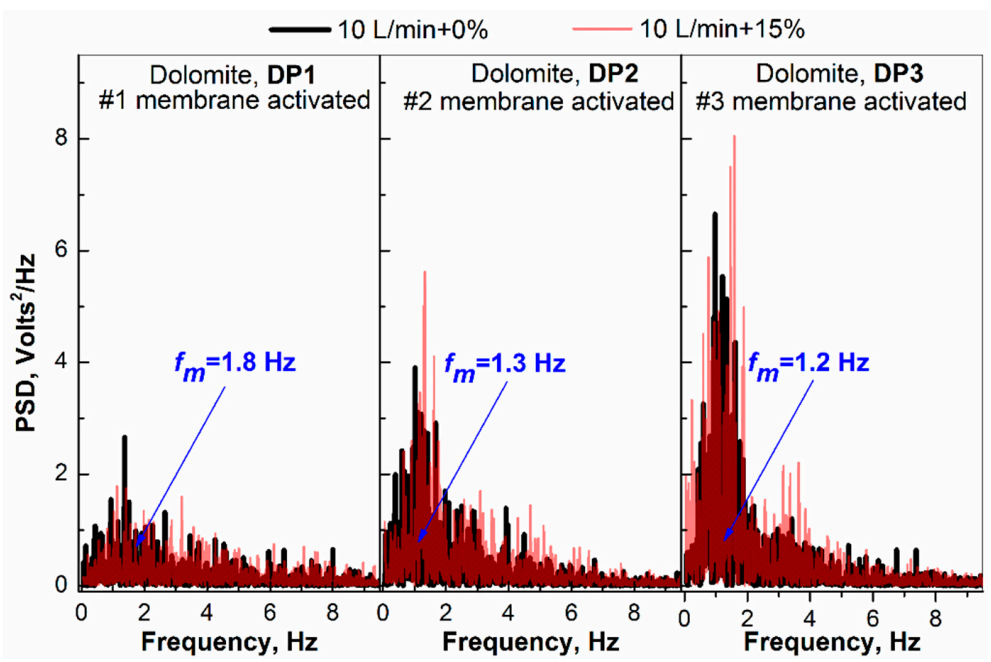

Figure 9. Comparison of the power spectrum of the differential pressure in the local bed section measured at gas addition and normal operational conditions. The other operational conditions were: $T=550{ }^{\circ} \mathrm{C}, P=0.1 \mathrm{MPa}, H_{\text {ini }}=1.28 \mathrm{~m}, U=0.09 \mathrm{~m} / \mathrm{s}$, addition rate $=1.5 \mathrm{~L} / \mathrm{min}$, calcined dolomite. 


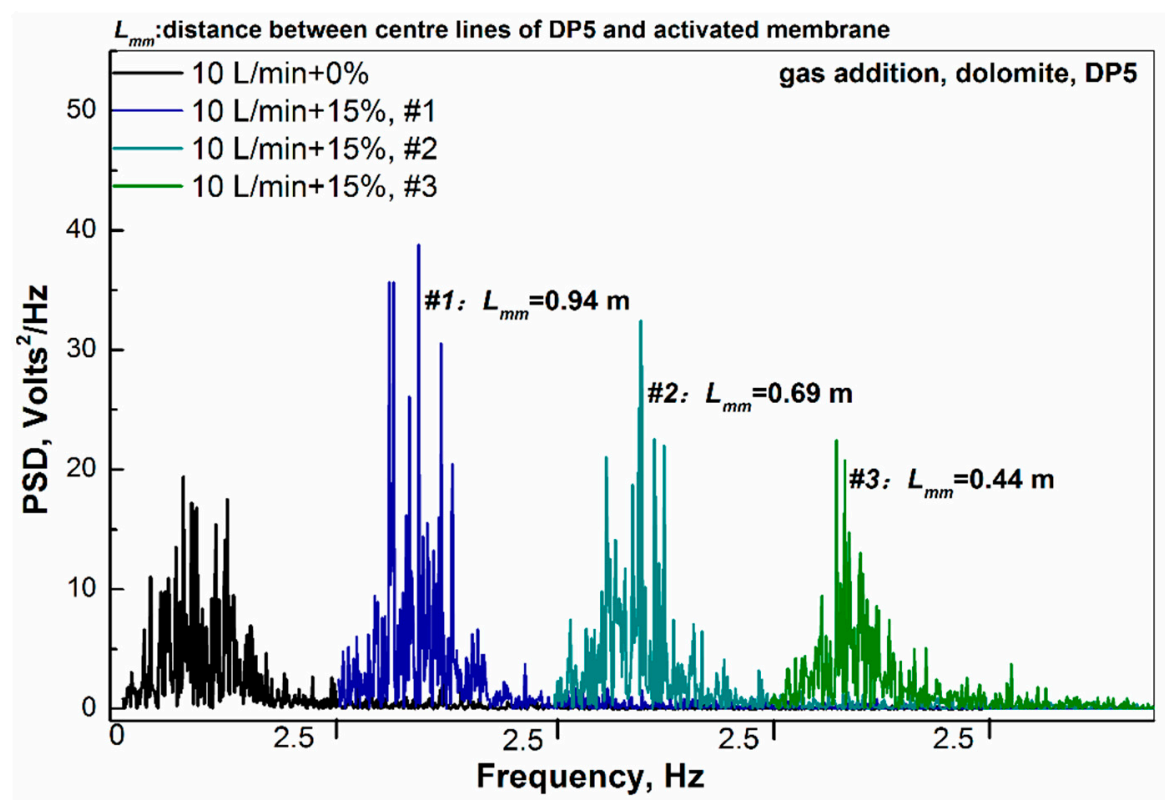

Figure 10. Effects of the gas addition location on the power spectra of the differential pressure measured at DP5. The other operational conditions: $T=550{ }^{\circ} \mathrm{C}, P=0.1 \mathrm{MPa}, H_{\text {in } \mathrm{i}}=1.28 \mathrm{~m}, U=0.09 \mathrm{~m} / \mathrm{s}$, addition rate $=1.5 \mathrm{~L} / \mathrm{min}$, calcined dolomite.

The energy fraction profiles of the different level sub-signals for the differential pressure signals measured at DP1-DP4, with individually activated membrane \#1-3 for gas addition, are displayed in Figure 11. In the local bed section, gas addition would slightly decrease the energy fraction of the D7 D6 $(0.39 \sim 1.56 \mathrm{~Hz})$ and increase the energy fraction of the D4 D2 $(3.125 \sim 25 \mathrm{~Hz})$ level sub-signals. No prominent change in the energy distribution of the differential pressure signals was observed in the upper bed sections far away from the addition location. The gas added at the local bed section through the porous membrane formed small bubbles, due to the increase of the excess gas in the bed. These small bubbles then merged with upward moving and growing bubbles, thus increasing the average size of large- and medium-sized bubbles in the upper bed sections. Zaabout et al. [37] found that gas addition through flat vertical membranes led to a larger number of smaller bubbles, but slightly reduced average bubble size. This discrepancy might be attributed to the adoption of a bed height that just covered the membrane plates in their experiments, so the emergence of small bubbles moving up was not observed. The occurrence of larger incrementation in the average bubble size at higher bed section can also be theoretically explained by an equation proposed by Darton et al. [36] as follows:

$$
d_{b}=0.54\left(U-U_{m f}\right)^{0.4}\left(h+4 \sqrt{\frac{A}{N_{o r}}}\right)^{0.8} g^{-0.2}
$$

The addition of gas resulted in higher superficial gas velocity, denoted as $U$ in Darton's equation, in the upper bed sections. Since the term of $U-U_{m f}$ in Equation (15) is larger under gas addition conditions, the increase of the average bubble size would also be larger than that under normal operational conditions at the same bed height $h$. 

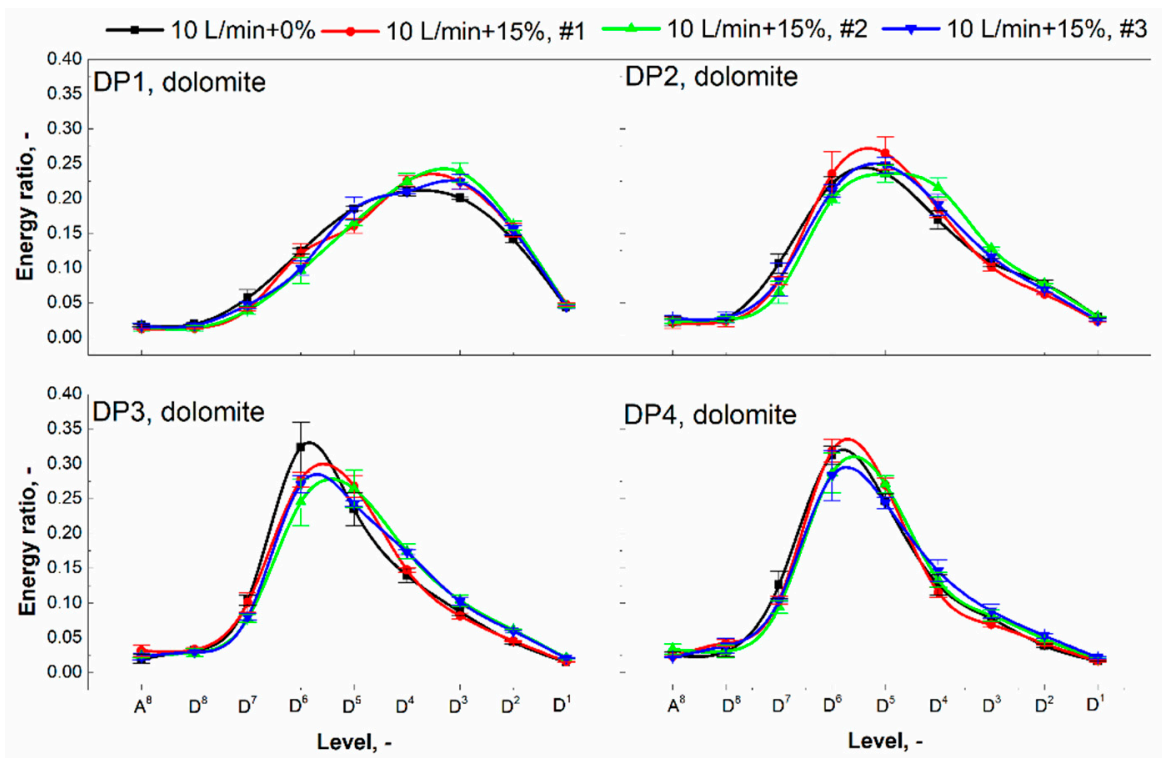

Figure 11. Effects of the gas addition location on the energy distribution profiles of multi-scale resolved differential pressure signals from DP1 DP4. The other operational conditions were: $T=550{ }^{\circ} \mathrm{C}, P=$ $0.1 \mathrm{MPa}, H_{\text {ini }}=1.28 \mathrm{~m}, U=0.09 \mathrm{~m} / \mathrm{s}$, addition rate $=1.5 \mathrm{~L} / \mathrm{min}$, calcined dolomite.

\subsection{Effects of Bed Material}

Using silica sand as bed material, the effects of gas extraction/addition through a porous-membrane on flow dynamics in the bed were also thoroughly investigated based on the differential pressure measurements. The results listed in Table 1 reveal that silica sand has a larger minimum fluidization velocity, of around $0.069 \mathrm{~m} / \mathrm{s}$, compared to calcined dolomite particles, mainly due to its larger density. A comparison of the evolution of the $\sigma_{r}$ along the height of the FBMR between the normal operation and gas extraction conditions is shown in Figure 12. When silica sand was used as bed material, the evolution of the $\sigma_{r}$ along the bed height during the gas addition tests was fairly consistent with the results obtained in experiments when dolomite particles are used, irrespective of whether operating with a single activated membrane or three activated membranes. Therefore, more attention had been paid to gas extraction conditions. As illustrated in Figure 12, whether at the inlet velocity of $0.09 \mathrm{~m} / \mathrm{s}$ or $0.14 \mathrm{~m} / \mathrm{s}$, the $\sigma_{r}$ of the differential pressure measured at DP1, DP2 and DP3 were increased by gas extraction, which was similar to observations made during tests using dolomite as bed material. However, at the bed sections DP4 and DP5, gas extraction led to a smaller $\sigma_{r}$ than that in the normal operation, contrary to experimental findings when dolomite particles were used.

The effects of gas extraction at specified locations on the hydrodynamics in different regions of the bed, using silica sand as bed material, were further investigated, as shown in Figure 13. With an extraction rate of $1.5 \mathrm{~L} / \mathrm{min}$, representing an extraction fraction of 15 and $10 \%$ for an inlet velocity of $0.09 \mathrm{~m} / \mathrm{s}$ and $0.14 \mathrm{~m} / \mathrm{s}$ accordingly, gas extraction would also cause an increase of the $\sigma_{r}$ of the differential pressure measured at the local bed section. Similar to tests with dolomite as bed material, the incrementation of the $\sigma_{r}$ also decreased as the distance of the measurement location from the extraction location increased. However, the decreasing rate was much faster than that of the tests using dolomite as bed material, which even caused the average bubble size to be smaller than that in normal operation at higher bed section, as indicated by green arrows in Figure 13. In addition, the divergence of the $\sigma_{r}$ between gas extraction and normal operation seemed to increase at higher bed sections. Wassie et al. [19] found that gas extraction through vertical membrane panels only caused larger equivalent bubble size when particles with a mean diameter of $196 \mu \mathrm{m}$ were used as a bed medium, while smaller average bubble size was observed when particles with a mean diameter of $500 \mu \mathrm{m}$ were used. They attributed the increase of the average bubble size, in cases when small particles were used, 
to the formation of densified zones, and suspected that the decrease in the average bubble size in the tests using larger particles was mainly caused by a decrease of the effective fluidizing velocity.

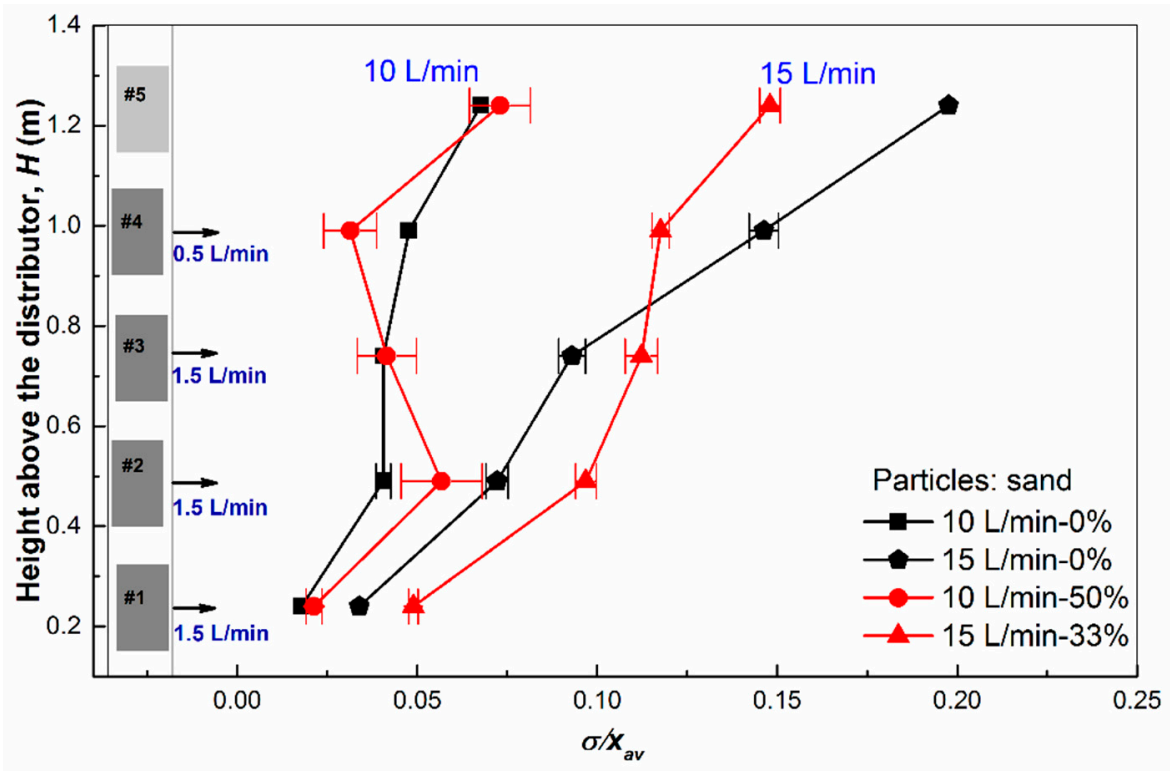

Figure 12. Effects of gas extraction through porous membranes on the $\sigma_{r}$ of the differential pressure signals. The other operation conditions were: $T=550{ }^{\circ} \mathrm{C}, P=0.1 \mathrm{MPa}, H_{\text {ini }}=1.28 \mathrm{~m}, U=0.09-0.14 \mathrm{~m} / \mathrm{s}$, silica sand.

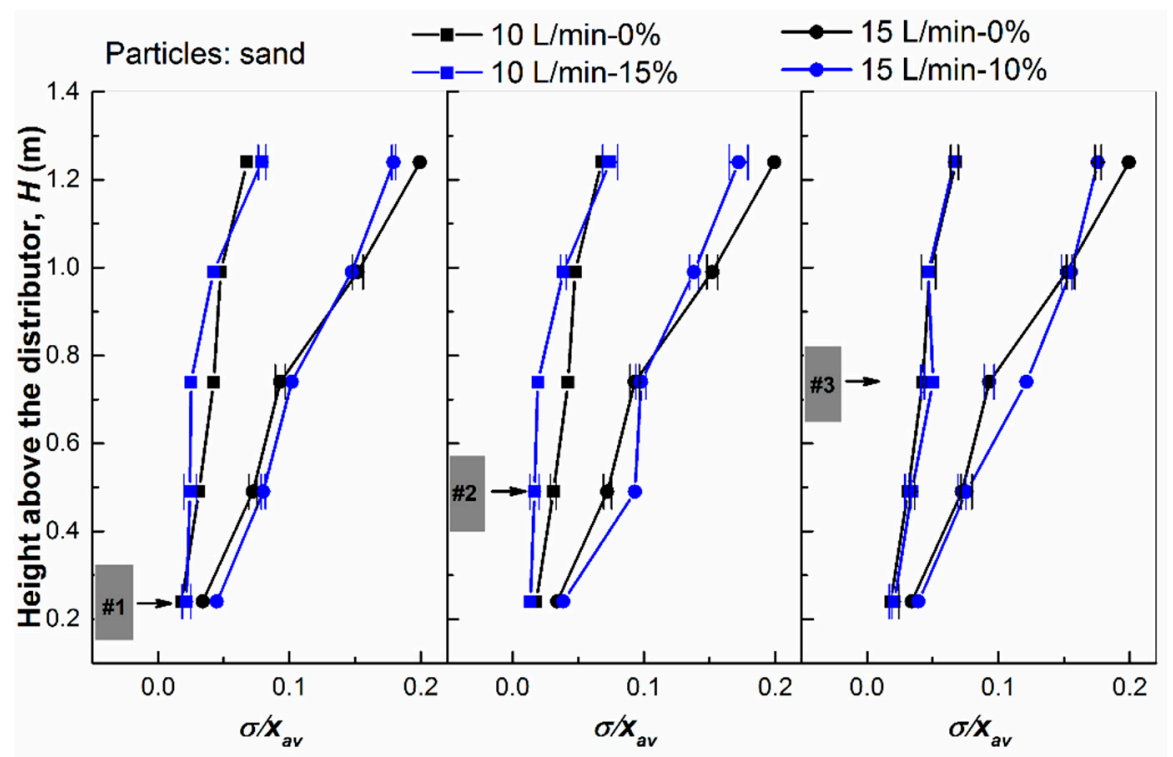

Figure 13. Effects of gas extraction on the $\sigma_{r}$ of the differential pressure signals with individually activated membrane \#1-3 for gas extraction. The other operation conditions were: $T=550{ }^{\circ} \mathrm{C}, P=$ $0.1 \mathrm{MPa}, H_{\text {ini }}=1.28 \mathrm{~m}, U=0.09 \sim 0.14 \mathrm{~m} / \mathrm{s}$, extraction rate $=1.5 \mathrm{~L} / \mathrm{min}$, silica sand.

The energy distribution profiles of the multi-scale resolved differential pressure signals measured in experiments using silica sand as bed material are shown in Figure 14. In the local bed section, gas extraction increased the energy fractions of the D7-D6 $(0.39 \sim 1.56 \mathrm{~Hz})$ level sub-signals, but decreased the energy fractions of the D4-D3 $(3.125 \sim 12.5 \mathrm{~Hz})$ level sub-signals of the differential pressure. This effect of gas extraction would also prolong into the upper bed sections, but decreases rapidly along the bed height. Thus, for differential pressure signals measured at DP4, with activated membranes \#1 or \#2, the energy ratios of the D6-D5 $(0.78 \sim 3.125 \mathrm{~Hz})$ level sub-signals were even lower than that in the normal operation. The power spectra of the differential pressure from the local bed section, displayed 
in Figure 15, show increased main peak intensity, and decreased high-frequency levels, compared with the normal operation. However, the power spectra of the differential pressure in the upper bed sections, for example, DP4 shows no remarkable variance, except for a slight decrease in the power density at low-frequency levels $(<1.2 \mathrm{~Hz})$.

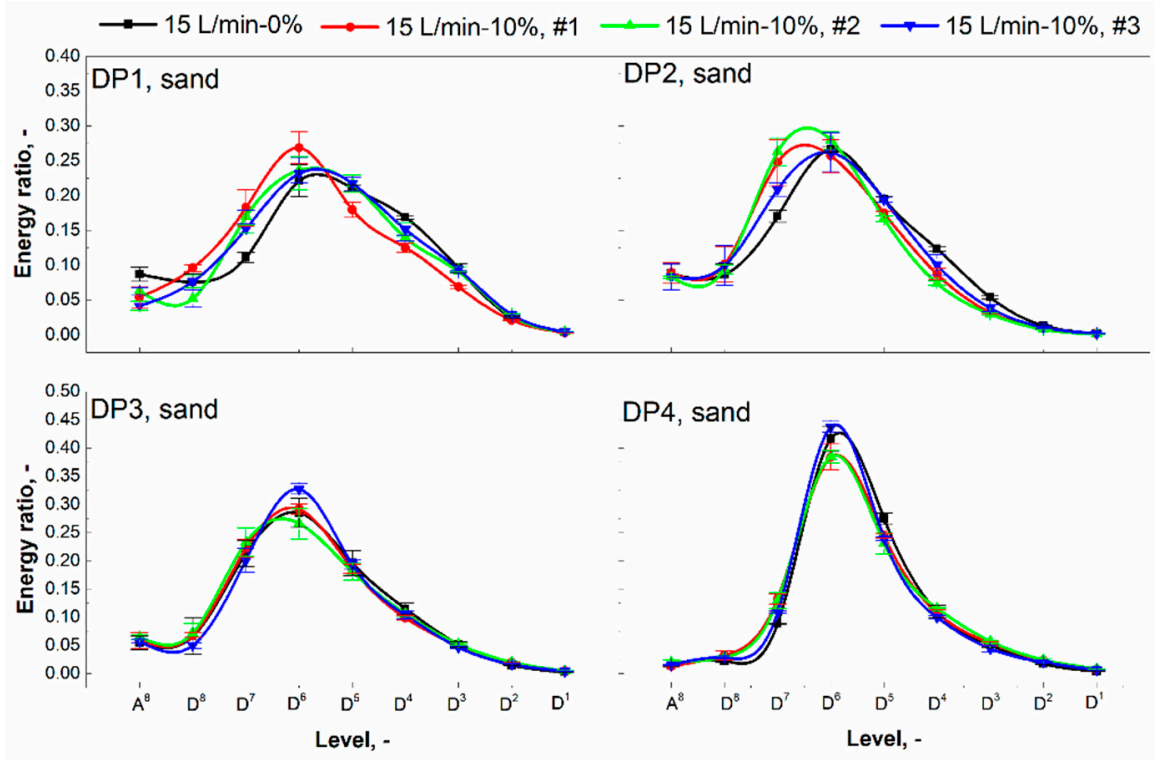

Figure 14. Effects of the gas extraction location on the energy distribution profiles of multi-scale resolved differential pressure from DP1 DP4. The other operational conditions were: $T=550{ }^{\circ} \mathrm{C}, P=$ $0.1 \mathrm{MPa}, H_{\text {ini }}=1.28 \mathrm{~m}, U=0.09 \mathrm{~m} / \mathrm{s}$, extraction rate $=1.5 \mathrm{~L} / \mathrm{min}$, silica sand.

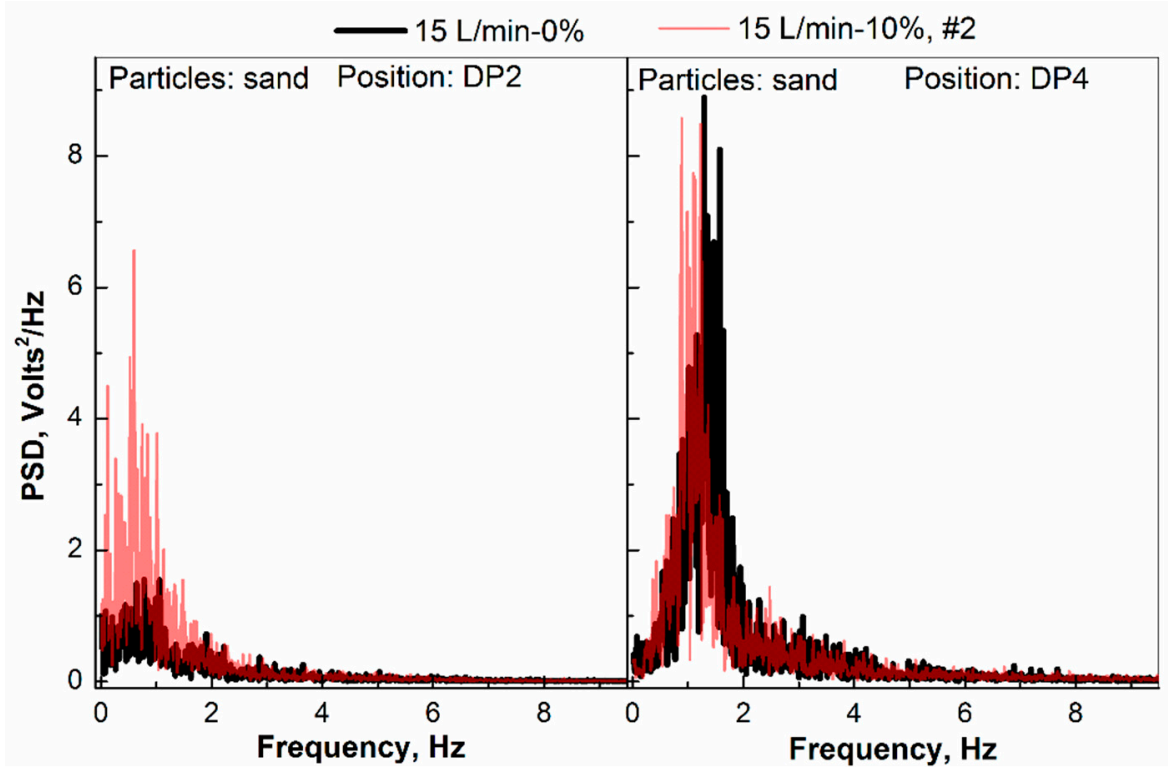

Figure 15. Power spectra of differential pressure measured at DP2 and DP4 with activated membrane \#2 for gas extraction. The other operational conditions were: $T=550{ }^{\circ} \mathrm{C}, P=0.1 \mathrm{MPa}, H_{\text {ini }}=1.28 \mathrm{~m}, \mathrm{U}$ $=0.09 \mathrm{~m} / \mathrm{s}$, addition rate $=1.5 \mathrm{~L} / \mathrm{min}$, silica sand.

Regardless of whether light or heavy particles (different from particle density) were used, gas extraction was found to increase the average bubble size in the local bed section, mainly due to the enhanced coalescence of small- and medium-sized bubbles in the fluidized bed. This enhancement effect could penetrate the bed sections above the active membrane panels, and decreased with the 
distance of the measurement locations from the extraction locations. However, the decreasing rate of this enhancement effect was much faster in the fluidized bed with particles of larger density.

\subsection{Mechanism Discussion}

Gas extraction/addition through porous membrane panels mainly affected the hydrodynamics in the bed via two distinct ways as follows: (1) by causing changes in the superficial gas velocity in the fluidized bed; (2) by exerting external forces on the particles/gas. The increase of the average bubble size caused by gas addition, especially in the bed section above the addition locations, was mainly attributed to the increase of the superficial gas velocity in the bed. Gas injected into the bed first formed small bubbles, and then merged with the bubbles passing by, so the increase of the average bubble size in the local bed section would not be so obvious due to the concurrent occurrence of small-bubble formation and bubble integration, as illustrated in Figure 16c.

(a)
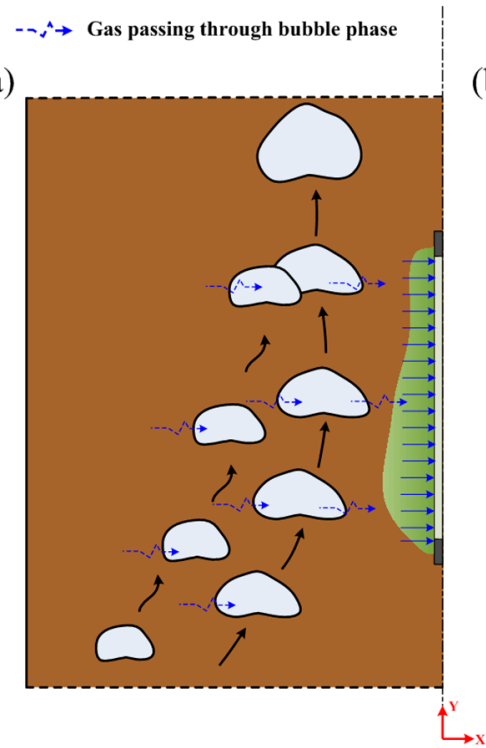

$\longrightarrow$ Gas extracted/added via membrane

(b)

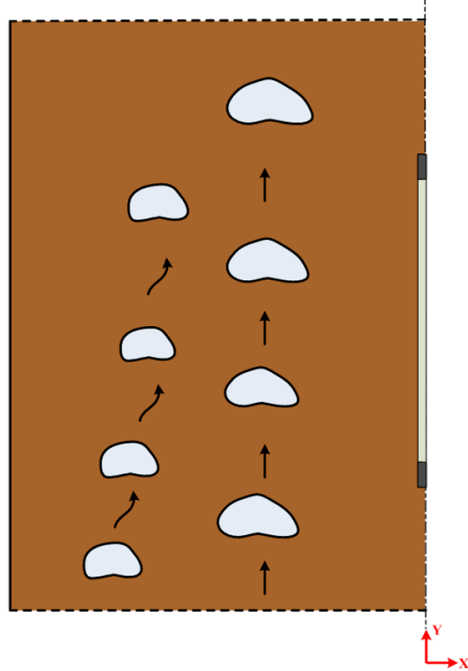

(c)
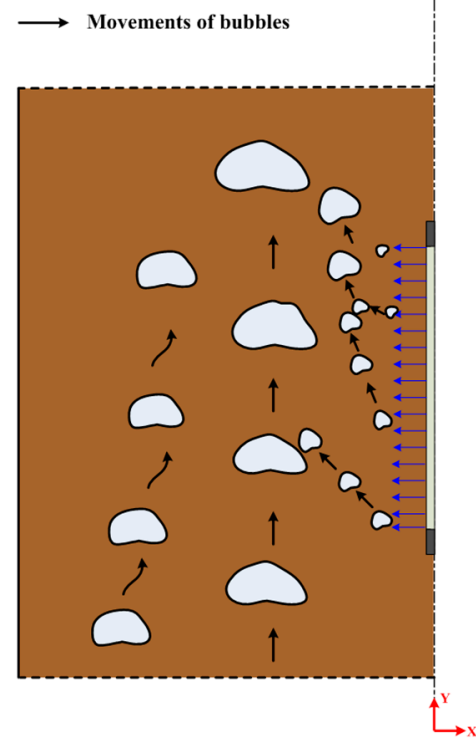

Figure 16. Illustration of the gas movement in the bed during (a) gas extraction; (b) normal operation; (c) gas addition.

Gas extraction through the porous membranes initially exerted external forces on the particles, which effectively balanced the gravity force with the assistance of the friction force of particles-particles and particles-membrane, so densified zones formed in the vicinity of the membranes [19]. The densified zones then reduced the space for bubbles to rise, thus enhancing bubble coalescence [16], which led to increase in the average bubble size. Besides, due to the narrowed cross-sectional area towards the upper bed section, the reduction of the effective fluidizing velocity caused by gas extraction would also be neutralized [19]. During the gas extraction experiment performed by de Jong et al. [16], the effects of gas extraction on particles movement were also found to disappear in the region above the extraction zones with a distance of $30 \mathrm{~cm} \mathrm{[16],} \mathrm{but} \mathrm{no} \mathrm{more} \mathrm{explanation} \mathrm{of} \mathrm{this} \mathrm{phenomenon}$ was provided.

As an additional reason, here we propose another explanation for the effects of gas extraction on the bubble properties in different regions of a FBMR. Generally, in a gas fluidized bed with a stable bubble phase, gas from the emulsion would enter into the "bubble" from the bottom and then escape from the top, to maintain a constant pressure in the "bubble", and also increase the rise of "bubbles". In the case of gas extraction, a lower pressure head is provided by the active membrane panels, which enhances the movement of the in-bed gas towards the active membranes. Consequently, the rise of the bubbles (especially for large bubbles because they usually have faster rising velocity) is slowed down, and the routes of bubble movements in the bed were "dragged" towards a similar 
direction, so the mergence of bubbles was greatly enhanced, which caused the average bubble size to increase, as shown in Figure 16a. In support of this explanation, a remarkable decrease of the bubble rise velocity was also observed at the middle and upper part of the local bed section during the gas extraction experiments performed by Wassie et al. [25]. This drag force exerted on gas essentially originated from the pressure gradients between the specified regions and the extraction location, and was maintained by a continuum of gas. Therefore, as the distance between the measurement location and extraction location increased, the enhancement of the effect of gas extraction on bubble coalescence is correspondingly reduced due to the increase of the flow resistance. When the effects from the decrease of the effective fluidizing velocity outperformed the enhancement from the "drag force", the average bubble size decreased continually, and eventually would be smaller than that in normal operations. The penetration length of this drag force can be estimated by the Ergun pressure drop equation [38], which can be expressed as:

$$
\frac{d P}{d L}=\frac{150 \mu}{d_{p}^{2}} \frac{(1-\varepsilon)^{2}}{\varepsilon^{3}} U+\frac{1.75 \rho}{d_{p}} \frac{(1-\varepsilon)}{\varepsilon^{3}} U|U|
$$

Here, $d P / d L$ represents the pressure drop per unit length, and $\varepsilon$ is the bed voidage. When silica sand particles (heavier particles) were used as bed medium, a smaller bed voidage was obtained, as indicated by the lower bed surface recorded in the experiments and smaller $\sigma_{r}$ was observed in Figure 12. According to Equation (16), larger $d P / d L$ would be achieved with smaller $\varepsilon$, which means that the pressure drop would be larger with the increase of the distance between the current position and the extraction location. Since the pressure head provided by gas extraction was constant, the penetration length of the drag force also decreased. This satisfactorily explained the divergence between the experimental results obtained in tests using calcined dolomite and silica sand as bed medium.

\section{Conclusions}

This work presented experimental studies on the effects of gas extraction/addition on the hydrodynamics in a pilot-scale FBMR, with vertical porous membrane panels installed in an orderly way along the height of the reactor. All permeation tests were mainly conducted at fluidization velocities of 0.09 and $0.14 \mathrm{~m} / \mathrm{s}$ at $550{ }^{\circ} \mathrm{C}$, with calcined dolomite and silica sand particles as bed medium, respectively. The differential pressure signals were measured at five different intervals, and then subjected to analysis, such as statistical analysis, power spectral and wavelet analysis. The effects of gas permeation on the gas-solid flow dynamics in different regions of the FBMR were thoroughly investigated.

The experimental results revealed that gas extraction via porous membrane panels caused the number of small- and medium-sized bubbles to decrease, and the average bubble size to increase in the local bed section, both of which were mainly attributed to the enhancement of the bubble coalescence. In bed sections above the activated membrane panel, the variation of the bubble properties during gas extraction was synergistically affected by the decrease of the effective fluidizing velocity and the drag force exerted by gas extraction on the gas movement. This drag force originated from the lower pressure head associated with gas extraction, and penetrated the bed through a gas continuum. As a result, it was attenuated with the increase of the distance from the extraction location, and quickly decreased in the fluidized bed with lower voidage. Accordingly, since calcined dolomite (light particles, larger bed voidage) was used as the bed material, the enhancement effect of gas extraction on bubble coalescence decreased gradually along the bed height. Thus, although the incrementation of the average bubble size was reduced, an average bubble size larger than that in normal operations was still observed. However, with silica sand particles (heavier particles, smaller bed voidage) as bed material, the attenuation of the enhancement effect from gas extraction was so fast that the average bubble size in the upper bed section was even smaller than that in normal operations. According to 
our experimental results, to avoid the occurrence of large bubbles the particles that readily achieve fluidization with lower voidage are preferred in a FBMR.

Irrespective of whether calcined dolomite or silica sand were employed as bed material, consistent results were obtained during the gas addition tests. Small bubbles would initially form in the local bed section and then merged with up-rising bubbles, so that a slight increase in the average bubble size was observed. Due to the increase of the superficial gas velocity in the upper bed sections, the growth of bubbles was effectively enhanced, and led to larger incrementation in the average bubble size at higher bed sections. Therefore, this suggests that the increase of the total fluidizing gas velocity should be taken into consideration during the design and operation of a gas fluidized bed reactor used in volume-increased processes. In this study, limited effects of the gas extraction/addition on the bubble properties were observed in bed sections below the activated membrane panels. This finding suggested that it was better not to position the membrane units in or below the fast-reacting zone when arranging multiple membranes in a large FBMR.

Author Contributions: Conceptualization, Y.C.; supervision, J.R.G.; data curation, Y.X. and R.P.; writing-original draft, C.B.

Funding: This work was supported by the Basic Research Programs of Science and Technology Commission Foundation of Jiangsu Province (BK20150196) and the Fundamental Research Funds for the Central Universities (2015XKMS058).

Conflicts of Interest: The authors declare no conflict of interest.

\section{References}

1. Roses, L.; Gallucci, F.; Manzolini, G.; Annaland, M.V. Experimental study of steam methane reforming in a Pd-based fluidized bed membrane reactor. Chem. Eng. J. 2013, 222, 307-320. [CrossRef]

2. Fernandez, E.; Helmi, A.; Coenen, K.; Melendez, J.; Viviente, J.L.; Tanaka, D.A.P.; Annaland, M.V.; Gallucci, F. Development of thin Pd-Ag supported membranes for fluidized bed membrane reactors including WGS related gases. Int. J. Hydrog. Energy 2015, 40, 3506-3519. [CrossRef]

3. Andrés, M.; Boyd, T.; Gulamhusein, A.; Comyn, N.; Lim, C.J.; Grace, J.R.; Shirasaki, Y.; Isamu, Y. Pure Hydrogen generation in a fluidized-bed membrane reactor: Experimental findings. Chem. Eng. Sci. 2008, 63, 2752-2762. [CrossRef]

4. Patil, C.S.; Annaland, M.V.; Kuipers, J.A.M. Fluidised bed membrane reactor for ultrapure hydrogen production via methane steam reforming: Experimental demonstration and model validation. Chem. Eng. Sci. 2007, 62, 2989-3007. [CrossRef]

5. Chen, Z.; Grace, J.R.; Lim, C.J.; Li, A. Experimental studies of pure hydrogen production in a commercialized fluidized-bed membrane reactor with SMR and ATR catalysts. Int. J. Hydrog. Energy 2007, 32, 2359-2366. [CrossRef]

6. Arratibel, A.; Tanaka, A.P.; Laso, I.; Annaland, M.V.; Gallucci, F. Development of Pd-based double-skinned membranes for hydrogen production in fluidized bed membrane reactors. J. Membr. Sci. 2018, 550, 536-544. [CrossRef]

7. Rakib, M.A.; Grace, J.R.; Lim, C.J.; Elnashaie, S.S.E.H. Steam reforming of heptane in a fluidized bed membrane reactor. J. Power Sources 2010, 195, 5749-5760. [CrossRef]

8. Gimeno, M.P.; Wu, Z.T.; Soler, J.; Herguido, J.; Li, K.; Menédez, M. Combination of a two-zone fluidized bed reactor with a Pd hollow fibre membrane for catalytic alkane dehydrogenation. Chem. Eng. J. 2009, 155, 298-303. [CrossRef]

9. Al-Sherehy, F.; Grace, J.R.; Adris, A.E.M. The influence of distributed reactant injection along the height of a fluidized bed reactor. Chem. Eng. Sci. 2005, 60, 7121-7130. [CrossRef]

10. Jao, S.; Arellanoi-Garcia, H.; Wozny, G. Oxidative coupling of methane in a fluidized bed reactor: Influence of feeding policy, hydrodynamics, and reactor geometry. Chem. Eng. J. 2011, 171, 255-271. [CrossRef]

11. Deshmukh, S.A.R.K.; Laverman, J.A.; Annaland, M.V.; Kuipers, J.A.M. Development of a membrane-assisted fluidized bed reactor. 2. Experimental demonstration and modeling for the partial oxidation of methanol. Ind. Eng. Chem. Res. 2005, 44, 5966-5976. [CrossRef] 
12. Christensen, D.; Nijenhuis, J.; van Ommen, J.R.; Coppens, M.O. Influence of distributed secondary gas injection on the performance of a bubbling fluidized-bed reactor. Ind. Eng. Chem. Res. 2008, 47, 3601-3618. [CrossRef]

13. Christensen, D.; Vervloet, D.; Nijenhuis, J.; van Wachem, B.G.M.; van Ommen, J.R.; Coppens, M.O. Insights in distributed secondary gas injection in a bubbling fluidized bed via discrete particle simulations. Powder Technol. 2008, 183, 454-466. [CrossRef]

14. Christensen, D.J.; Nijenhuis, J.; van Ommen, J.R.; Coppens, M.O. Residence times in fluidized beds with secondary gas injection. Powder Technol. 2008, 180, 321-331. [CrossRef]

15. Mleczko, L.; Ostrowski, T.; Wurzel, T. A fluidized-bed membrane reactor for the catalytic partial oxidation of methane to synthesis gas. Chem. Eng. Sci. 1996, 51, 3187-3192. [CrossRef]

16. De Jong, J.F.; Annaland, M.V.; Kuipers, J.A.M. Experimental study on the effects of gas permeation through flat membranes on the hydrodynamics in membrane-assisted fluidized beds. Chem. Eng. Sci. 2011, 66, 2398-2408. [CrossRef]

17. Dang, N.T.Y.; Gallucci, F.; Annaland, M.V. Micro-structured fluidized bed membrane reactor: Solids circulation and densified zones distribution. Chem. Eng. J. 2014, 239, 42-52. [CrossRef]

18. Wassie, S.A.; Cloete, S.; Zaabout, A.; Gallucci, F.; Annaland, M.V.; Amini, S. Experimental investigation on the generic effects of gas permeation through flat vertical membranes. Powder Technol. 2017, 316, 207-217. [CrossRef]

19. Wassie, S.A.; Zaabout, A.; Gallucci, F.; Cloete, S.; Annaland, M.V.; Amini, S. Detecting densified zone formation in membrane-assisted fluidized bed reactors through pressure measurements. Chem. Eng. J. 2017, 308, 1154-1164. [CrossRef]

20. Helmi, A.; Wagner, E.C.; Gallucci, F.; Annaland, M.V.; van Ommen, J.R.; Mudde, R.F. On the hydrodynamics of membrane assisted fluidized bed reactors using X-ray analysis. Chem. Eng. Process. 2017, 122, 508-522. [CrossRef]

21. De Jong, J.F.; Annaland, M.V.; Kuipers, J.A.M. Experimental study on the hydrodynamic effects gas permeation through horizontal membrane tubes in fluidized beds. Powder Technol. 2013, 241, 74-84. [CrossRef]

22. Julián, I.; Herguido, J.; Menéndez, M. Gas permeation effect on the two-section two-zone fluidized bed membrane reactor (TS-TZFBMR) fluid dynamics: A CFD simulation study. Chem. Eng. J. 2016, 305, 201-211. [CrossRef]

23. Dang, T.Y.N.; Gallucci, F.; Annaland, M.V. Gas back-mixing study in a membrane-assisted micro-structured fluidized bed. Chem. Eng. Sci. 2014, 108, 194-202. [CrossRef]

24. Min, J.; Drake, J.B.; Heindel, T.; Fox, R. Experimental validation of CFD simulation of a Lab-scale fluidized-bed reactor with and without side-gas injection. AIChE J. 2010, 56, 1434-1445. [CrossRef]

25. Wassie, S.A.; Gallucci, F.; Cloete, S.; Zaabout, A.; Annaland, M.V.; Amini, S. The effect of gas permeation through vertical membrane on chemical switching reforming (CSR) reactor performance. Int. J. Hydrog. Energy 2016, 41, 8640-8655. [CrossRef]

26. Gómez-Hernández, J.; Sánchez-Prieto, J.; Briongos, J.V.; Santana, D. Wide band energy analysis of fluidized bed pressure fluctuation signals using a frequency division method. Chem. Eng. Sci. 2014, 105, 92-103. [CrossRef]

27. Bi, H.T.; Grace, J.R.; Zhu, J. Propagation of pressure waves and forced oscillations in gas-solid fluidized beds and their influence on diagnostics of local hydrodynamics. Powder Technol. 1995, 82, 239-253. [CrossRef]

28. Van der Schaaf, J.; Schouten, J.C.; van den Bleek, C.M. Origin, propagation and attenuation of pressure waves in gas-solid fluidized beds. Powder Technol. 1998, 95, 220-233. [CrossRef]

29. Chen, Y.; Lim, C.J.; Grace, J.R.; Zhang, J.; Zhao, Y. Characterization of pressure fluctuations from a gas-solid fluidized bed by structure density function analysis. Chem. Eng. Sci. 2015, 129, 156-167. [CrossRef]

30. Chen, Y.; Chen, W.; Grace, J.R.; Zhao, Y.; Zhang, J.; Li, Y. Direct resolution of differential pressure fluctuations to characterize multi-scale dynamics in a gas fluidized bed. Int. J. Multiph. Flow 2016, 85, 380-394. [CrossRef]

31. Rakib, M.A.; Grace, J.R.; Lim, C.J.; Elnashaie, S.S.E.H. Steam reforming of propane in a fluidized bed membrane reactor for hydrogen production. Int. J. Hydrog. Energy 2010, 35, 6276-6299. [CrossRef]

32. Peters, T.A.; Stange, M.; Bredense, R. On the high pressure performance of thin supported Pd- $23 \% \mathrm{Ag}$ membrane-evidence of ultrahigh hydrogen flux after air treatment. J. Membr. Sci. 2011, 378, 28-34. [CrossRef] 
33. Johnsson, F.; Zijerveld, R.C.; Schouten, J.C.; van den Bleek, C.M.; Lecker, B. Characterization of fluidization regimes by time-series analysis of pressure fluctuations. Int. J. Multiph. Flow 2000, 26, 663-715. [CrossRef]

34. Zhao, G.B.; Yang, Y.R. Multiscale resolution of fluidized-bed pressure fluctuations. AIChE J. 2003, 49, 869-882. [CrossRef]

35. Wu, B.; Kantzas, A.; Bellehuneur, C.T.; He, Z.; Kryuchkov, S. Multiresolution analysis of pressure fluctuations in a gas-solids fluidized bed: Application to glass beads and polyethylene powder systems. Chem. Eng. J. 2007, 131, 23-33. [CrossRef]

36. Darton, R.C.; Lanauuze, R.D.; Davidson, J.F.; Harrison, D. Bubble growth due to coalescence in fluidized-beds. Trans. Inst. Chem. Eng. 1977, 55, 274-280.

37. Zaabout, A.; Bernus, A.; Cloete, S.; Amini, S. The effect of gas addition on bubble dynamics in a fluidized bed with flat vertical membranes. Chem. Eng. J. 2018, 344, 71-85. [CrossRef]

38. Ergun, S. Fluid flow through packed columns. Chem. Eng. Prog. 1952, 48, 89-94.

2018 by the authors. Licensee MDPI, Basel, Switzerland. This article is an open access article distributed under the terms and conditions of the Creative Commons Attribution (CC BY) license (http://creativecommons.org/licenses/by/4.0/). 